\title{
Altered T-cell subset repertoire affects treatment outcome of patients with myelofibrosis
}

\author{
Ivo Veletic, ${ }^{1, *}$ Sanja Prijic,,${ }^{1,2, *}$ Taghi Manshouri, ${ }^{1}$ Graciela M. Nogueras-Gonzalez, ${ }^{3}$ \\ Srdan Verstovsek, ${ }^{1}$ and Zeev Estrov ${ }^{1}$ \\ ${ }^{1}$ Department of Leukemia, The University of Texas MD Anderson Cancer Center, \\ Houston, TX, USA; ${ }^{2}$ Clinical Department of Laboratory Diagnostics, University Hospital \\ Center Zagreb, Zagreb, Croatia and ${ }^{3}$ Department of Biostatistics, The University of Texas \\ MD Anderson Cancer Center, Houston, TX, USA
}

Haematologica 2021

Volume 106(9):2384-2396

*IV and SP contributed equally to the study as co-first authors.

\section{ABSTRACT}

P henotypic characterization of $\mathrm{T}$ cells in myelofibrosis is intriguing because of increased inflammation, markedly elevated pro-inflammatory cytokines, and altered distribution of T-cell subsets. Constitutive activation of Janus kinase-2 (JAK2) in the majority of patients with myelofibrosis contributes to the expression of the programmed cell death protein-1 (PD1) and T-cell exhaustion. We wondered whether T-cell activation affects treatment outcome of patients with myelofibrosis and sought to determine whether the JAK1/2 inhibitor ruxolitinib affects the activation of T-cell subsets. T cells from 47 myelofibrosis patients were analyzed and the percentages of either helper $\left(\mathrm{CD} 4^{+}\right)$or cytotoxic $\left(\mathrm{CD}^{+}\right)$naïve, central memory, effector memory, or effector $\mathrm{T}$ cells; and fractions of PD1-expressing cells in each subset were assessed. Higher numbers of $\mathrm{T}$ cells co-expressing CD4/PD1 and CD8/PD1 were found in myelofibrosis patients than in healthy controls $(n=28)$, and the $T$ cells were significantly skewed toward an effector phenotype in both $\mathrm{CD}^{+}$and $\mathrm{CD}^{+}$subsets, consistent with a shift from a quiescent to an activated state. Over the course of ruxolitinib treatment, the distribution of aberrant T-cell subsets significantly reversed towards resting cell phenotypes. $\mathrm{CD}^{+}$and $\mathrm{CD}^{+}$subsets at baseline correlated with monocyte and platelet counts, and their $\mathrm{PD}^{+}$fractions correlated with leukocyte counts and spleen size. Low numbers of $\mathrm{PD} 1^{+} / \mathrm{CD} 4^{+}$and $\mathrm{PD} 1^{+} / \mathrm{CD} 8^{+}$cells were associated with complete resolution of palpable splenomegaly and improved survival rate, suggesting that low levels of exhausted $\mathrm{T}$ cells confer a favorable response to ruxolitinib treatment.

Received: February 6, 2020.

Accepted: July 16, 2020.

Pre-published: July 30, 2020.

https://doi.org/10.3324/haematol.2020.249441

(C)2021 Ferrata Storti Foundation

Material published in Haematologica is covered by copyright. All rights are reserved to the Ferrata Storti Foundation. Use of published material is allowed under the following terms and conditions:

https://creativecommons.org/licenses/by-nc/4.0/legalcode. Copies of published material are allowed for personal or internal use. Sharing published material for non-commercial purposes is subject to the following conditions:

https://creativecommons. org//icenses/by-nc/4.0/legalcode, sect. 3. Reproducing and sharing published material for commercial purposes is not allowed without permission in writing from the publisher.

\section{Introduction}

Primary or secondary myelofibrosis $(\mathrm{MF})$ is characterized by a significant immune deregulation. ${ }^{1,2}$ In the vast majority of patients with MF, Janus kinase (JAK)-2 is constitutively activated. ${ }^{3}$ As a result, MF neoplastic cells produce high levels of inflammatory cytokines and pentraxins that contribute to the induction of progressive bone marrow (BM) fibrosis, debilitating constitutional symptoms, and poor prognosis in MF patients. ${ }^{4,5}$ Cytokines, such as interleukin (IL)-1, IL-6 and IL8 , modulate T-cell activation and immune function through the activation of JAK2 and its downstream signal transducer and activator of transcription (STAT) pathways. ${ }^{6,7}$ The JAK1/2 inhibitor ruxolitinib alleviates constitutional symptoms in MF patients, primarily by profound suppression of inflammation. ${ }^{8}$ Although it is known that JAK-STAT signaling modifies T-helper cell activity and inflammatory responses and JAK1/2 inhibition impairs the cytotoxic function of $\mathrm{T}$ cells in vitro, ${ }^{11}$ the effects of aberrant JAK2 signaling and its modulation of $\mathrm{T}$ cells in patients with $M F$ remain elusive.

A few recent studies showed increased T-cell response to neo-antigens in patients with myeloproliferative neoplasms. ${ }^{12-15}$ However, persistent tumorinduced activation prompts $T$ cells to enter a dysfunctional state, referred to as $T$ - 
cell exhaustion. ${ }^{16}$ Programmed cell death protein 1 (PD1) is an essential T-cell checkpoint receptor that is overexpressed as T cells undergo persistent activation, thus providing a pathway to control T-cell response. ${ }^{17}$ In several neoplasms, PD1 was found to play a critical role in regulating T-cell function. ${ }^{18}$ Recent studies showed that myeloid cells from subjects with myeloproliferative neoplasms express high levels of PD1 ligand 1 (PDL1), ${ }^{19,20}$ and that the overexpression of PDL1 is induced by constitutively activated JAK2. ${ }^{19}$ Elevated levels of PDL1 on tumor cells frequently correlate with increased JAK2 activity, ${ }^{21}$ and high PDL1 expression confers sensitivity to anti-PD1 therapy. ${ }^{22}$ Conversely, decreased PDL1 levels were detected in rare solid tumors harboring loss-of-function JAK2 mutations, and have been associated with poor outcomes of PD1/PDL1 blockade therapy. ${ }^{23}$

Because JAK2 is constitutively activated in MF, we sought to determine whether the T-cell activation status is altered in MF patients and whether and how it is affected by JAK inhibitor therapy. We also sought to elucidate the relationship between T-cell activation and exhaustion given the implications of this association in treating MF patients, developing immune-harnessing MF strategies, and rationally guiding clinical trials. To achieve these goals, we systematically analyzed the activation status of peripheral T-cell subsets of patients with MF at baseline and over the course of treatment with ruxolitinib. Furthermore, we tested the association of PD1-co-expressing helper $\left(\mathrm{CD}^{+}\right)$ and cytotoxic $\left(\mathrm{CD} 8^{+}\right) \mathrm{T}$-cell subpopulations with disease progression and assessed the effect of $\mathrm{PD} 1^{+} \mathrm{T}$-cell fractions on the clinical outcome of MF patients.

\section{Methods}

\section{Specimen assessed in this study}

We obtained corresponding $\mathrm{BM}$ and peripheral blood (PB) specimens from 47 patients with MF who were enrolled in a phase I/II clinical trial of ruxolitinib at the University of Texas MD Anderson Cancer Center (MDACC) (ClinicalTrials.gov identifier, NCT00509899) between June 2007 and April 2015. ${ }^{24}$ Specimens were collected prior to treatment and for up to 7 years after treatment, once Institutional Review Board (IRB)approved informed consent had been obtained. The patients were diagnosed with primary MF, post-essential thrombocythemia MF or post-polycythemia vera MF; the diagnoses were established in accordance with the 2008 World Health Organization classification..$^{25}$ The patients did not receive antineoplastic medications for at least 14 days before starting treatment. Ruxolitinib was administered orally (10-25 mg twice a day or 50-200 mg once a day) according to the clinical trial protocol, which was designed to assess the efficacy and safety of ruxolitinib. For control studies, PB specimens were obtained from 28 healthy individuals (13 males and 15 females) agematched with MF patients. The clinical and laboratory research was conducted in accordance with the Declaration of Helsinki and approved by the MDACC IRB1 committee (protocol identities LAB01-473 and LAB05-0321).

\section{Cell fractionation and immunostaining}

To detect and quantify cell surface proteins, we performed multiparameter flow cytometry analyses of low-density cells that were previously stored in dimethylsulfoxide. Briefly, BM and $\mathrm{PB}$ specimens were collected into Vacutainer tubes contain-
A

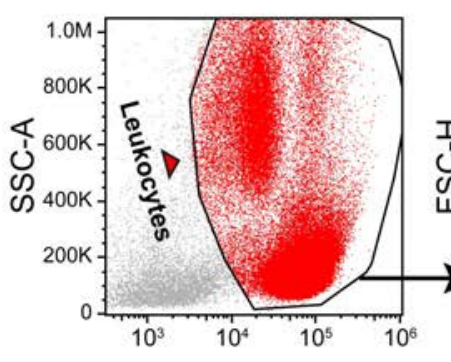

CD45 PE-CF594

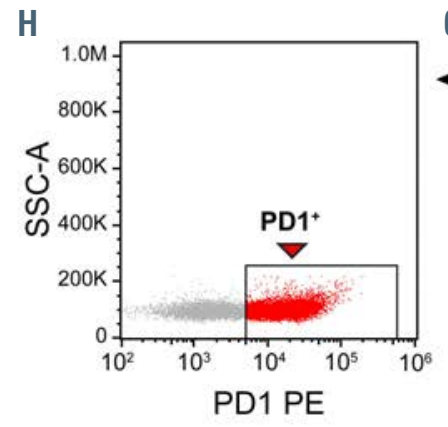

B

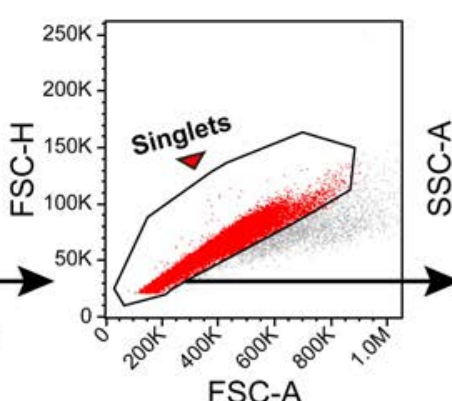

C

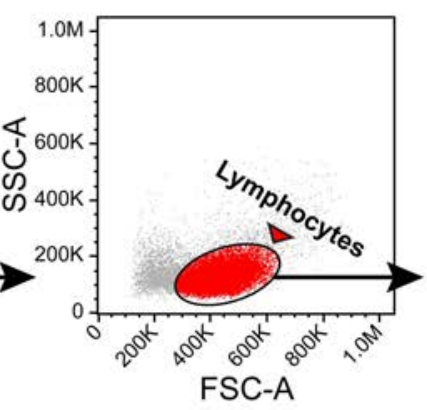

D

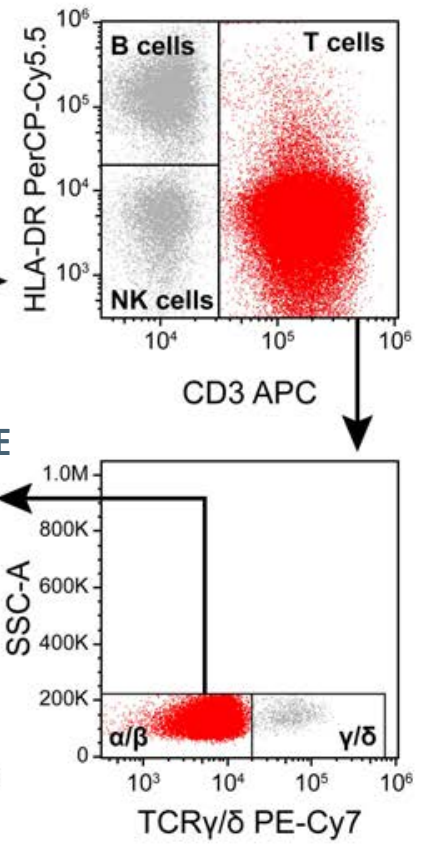

Figure 1. T-cell subset gating strategy. Representative dot plots demonstrating the flow cytometry gating strategy that was used to analyze individual T-cell subsets. (A) First, leukocytes were isolated based on CD45 (PE-CF594) positivity and side scatter area (SSC-A). (B) Singlets were then gated using forward scatter area (FSCA) and height (FSC-H). (C) Within the singlet leukocyte population, lymphocytes were defined based on FSC-A and SSC-A parameters. (D) Subsequently, T cells were separated from B cells and natural killer (NK) cells based on positivity for CD3 (APC) and HLA-DR (PerCP-Cy5.5). (E) Gamma delta ( $\gamma / \delta$ ) T cells were excluded from further analysis using T-cell receptor gamma delta (TCRY/ $\delta$; PE-Cy7) and SSC-A. (F) Alpha beta $(\alpha / \beta)$ T cells were further gated to differentiate between CD4 ${ }^{+}$and

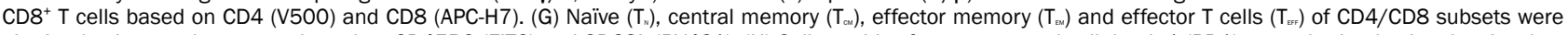
obtained using quadrant gates based on CD45RO (FITC) and CD62L (BV421). (H) Cells positive for programmed cell death 1 (PD1) were obtained using density plots for PD1 (PE) with the corresponding isotype control as reference. Red dots depict T cells within each of the previously defined gates; only the CD4 ${ }^{+}$subset is displayed in $(\mathrm{G})$ and $(\mathrm{H})$. 
ing sodium heparin (BD, Franklin Lakes, NJ, USA). Low-density cells were fractionated using Ficoll-Histopaque 1077 (SigmaAldrich, St. Louis, MO, USA), washed in phosphate-buffered saline (Invitrogen, Carlsbad, CA, USA), spun down, dissolved in $10 \%$ dimethylsulfoxide solution (Sigma-Aldrich, St. Louis, MO, USA) supplemented with fetal bovine serum (Invitrogen, Carlsbad, CA, USA), and frozen in liquid nitrogen. Prior to flow cytometry analysis, cells were thawed, washed and re-suspended in fetal bovine serum. After trypan blue viability assessment, live cells $\left(10^{6}\right)$ were incubated with the appropriate antibodies or their corresponding isotype controls, and their cell surface protein expression was assessed using the Gallios multichannel flow cytometer (Beckman Coulter, Brea, CA, USA). The antibodies used and their isotype controls are listed in Online Supplementary Table S1.

\section{Flow cytometry analysis of $\mathrm{T}$ cells}

A universal gating strategy was applied to identify individual T-cell subsets. Singlet lymphocytes in the $\mathrm{CD} 45^{+}$cell population were identified based on size and lack of granularity (Figure 1AC). Subsequently, $T$ cells were gated by using anti-CD3 and antiHLA-DR antibodies and further subdivided using anti-TCR $\gamma / \delta$ antibodies (Figure $1 \mathrm{D}$ and $\mathrm{E}$ ). The $\mathrm{CD}^{+}$and $\mathrm{CD} 8^{+}$subpopulations of the $\alpha / \beta^{+} T$ cells were further separated into naive $\left(T_{N}\right)$, central memory $\left(T_{c m}\right)$, effector $\left(T_{m}\right)$, and effector memory $\left(T_{\mathrm{m}}\right)$ subsets, using anti-CD62L and anti-CD45RO antibodies (Figure $1 \mathrm{~F}$ and $\mathrm{G})$. The percent of $\mathrm{PD} 1^{+} \mathrm{T}$ cells was assessed in each subset (Figure $1 \mathrm{H})$. All flow cytometry data were analyzed using FlowJo software v10.5 (Treestar, San Carlos, CA, USA).

\section{Statistical analyses}

The Student $t$-test was used to assess whether T-cell subsets of normal individuals were significantly different from those of patients with MF. A paired $t$-test was used to determine whether ruxolitinib treatment significantly affected T-cell subset distribution. A linear mixed-effects model with repeated measures was developed to determine whether there were differences in T-cell subsets at sequential time-points. In order to correct for clinical response or progression over time, several model specifications that included clinical variables obtained at the time of sample collection were compared using mean Akaike and Bayesian information criteria and R-squared values, and the best performing model was selected for use in the longitudinal analyses. Significance of overall change in time and each predictor were assessed using the Kenward-Roger adjusted F-test. Correlations between continuous clinical variables and T-cell subsets were assessed using the Pearson coefficient and between-group differences were calculated using the Welch $t$-test. The percentage of each $\mathrm{T}$-cell subset was dichotomized into high and low groups using the optimal cutoff value of maximally selected rank statistics. The patients' overall survival was estimated by the KaplanMeier method and a log-rank test was used to compare the survival probabilities. A univariate Cox proportional hazard regression model was fitted to assess the association between clinical variables and overall survival. To assess the predictive value of T-cell subsets, a multivariate Cox proportional hazard model was applied, adjusted for the clinical variables that were found to be significant in the univariate analyses. The Wald test was used to assess the significance of each covariate in Cox models. Statistical analysis was performed using Stata/SE v15.1 (Stata Corp, College Station, TX, USA) and R v3.6.3 (R Foundation for Statistical Computing, Vienna, Austria) statistical software with tidyverse v1.3.0, lme4 v1.1-23, pbkrtest v0.4-8.6, and survival v3.1-8 packages. Graphs were created using GraphPad Prism v7.03 (GraphPad Software, La Jolla, CA, USA) and R packages corrplot v0.84, ggplot2 v3.3.0, ggpubr v0.2.5, ggeffects v0.14.3, and survminer v0.4.6.

\section{Data sharing statement}

De-identified original data are available from the corresponding author (zestrov@mdanderson.org).

\section{Results}

\section{Clinical features of myelofibrosis patients and out- come of ruxolitinib treatment}

To evaluate the distribution and characteristics of T-cell subsets in patients with MF, we analyzed $\mathrm{PB}$ and BM specimens of $47 \mathrm{MF}$ patients (27 with PMF, 13 with post-polycythemia vera MF, and 7 with post-essential thrombocythemia MF) and 28 agematched healthy controls (Online Supplementary Table S2). The median daily dose of ruxolitinib was $50 \mathrm{mg}$ (range, 20-200 mg) at the start of the clinical trial. The dose was reduced because of anemia and/or thrombocytopenia in seven patients. The median duration of treatment was 38.9 months. Among the patients who had their specimens analyzed in this study, two discontinued treatment because of myelosuppression and four because of transformation to acute myeloid leukemia. Infection (pneumonia and/or sepsis) was the cause of death in five of the 16 $(31.3 \%)$ patients who died while on trial.

\section{Analysis of myelofibrosis patients' T-cell subsets}

The percentages of $\mathrm{CD}^{+}$and $\mathrm{CD}^{+}$cells and their $\mathrm{T}_{\mathrm{N}}, \mathrm{T}_{\mathrm{c}}, \mathrm{T}_{\mathrm{H}}$, and $T_{\mathrm{DM}}$ subsets were assessed in BM or PB specimens from $41 \mathrm{MF}$ patients and 28 healthy individuals. Because analyses of T-cell subsets using $P B(n=35)$ and $B M(n=16)$ specimens from the same MF patients revealed similar results (Online Supplementary Figure S3), we have not presented the data separately. Whereas $\mathrm{CD}^{+}$and $\mathrm{CD} 8^{+}$ cell distributions in MF patients were not different from those in healthy individuals (Figure 2Ai and Bi), marked differences were found in both CD4-derived (Figure 2Aii and Bii) and CD8-derived (Figure 2Aiii and Biii) T-cell subsets. We detected a 2.93-fold and a 3.45 -fold $\left(P<0.001\right.$ for both) reduction in the number of $T_{N}$ cells, and a 3.45 -fold and a 4.03 -fold ( $P<0.001$ for both) reduction in $\mathrm{T}_{\mathrm{c}}$ cells within the $\mathrm{CD}^{+}$and $\mathrm{CD}^{+}$cell subsets, respectively, in MF-derived T cells as compared to normal controls. Conversely, we detected an increase in the number of $\mathrm{T}_{\mathrm{BF}}$ cells within both $\mathrm{CD}^{+}$and $\mathrm{CD} 8^{+}$cell subsets (mean fold changes, 2.75 and 1.86 , respectively; $P<0.001$ for both), and in the number of $\mathrm{T}_{\mathrm{Bn}}$ cells within the $\mathrm{CD}^{+}$cell fraction (mean fold change, $1.51 ; P=0.005$ ) but not within the $\mathrm{CD} 8^{+}$cell fraction. Whereas $\mathrm{CD}^{+}$and $\mathrm{CD}^{+}$resting subsets $\left(\mathrm{T}_{\mathrm{N}}\right.$ and $\left.\mathrm{T}_{\mathrm{c}}\right)$ in $\mathrm{MF}$ patients correlated significantly and positively with one another, two effector subsets $\left(T_{\mathrm{B}}\right.$ and $T_{\mathrm{HI}}$ ) exhibited negative correlation between both one another and the resting subsets (Figure 2C), indicating that one effector population prevails within each patient's CD4 or CD8 subset. Overall, the increase in effector T-cell phenotype suggests that in patients with MF T cells shift from a quiescent to an activated state. Compared to $\mathrm{CD}^{+}, \mathrm{MF} \mathrm{CD} 8^{+} \mathrm{T}$ cells shift more towards a terminally activated state, suggesting a predominant effector-mediated cytotoxic response in MF.

\section{Long-term effects of ruxolitinib treatment on T-cell subsets}

Because treatment with ruxolitinib reduces plasma levels of cytokines and chemokines and significantly reduces spleen size in most MF patients, ${ }^{24,26,27}$ we sought to assess the effect of ruxolitinib treatment on the distribution of T-cell subsets. Analysis of the corresponding $\mathrm{PB}$ or $\mathrm{BM}$ specimens obtained from $25 \mathrm{MF}$ patients before and during ruxolitinib treatment demonstrated an overall shift towards a $\mathrm{CD}^{+}$phenotype over the course of 
MF

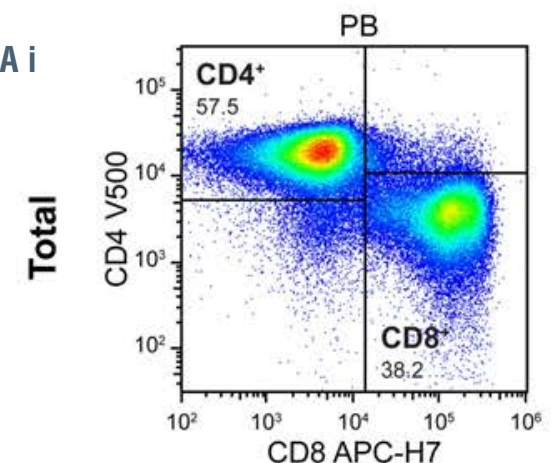

ii

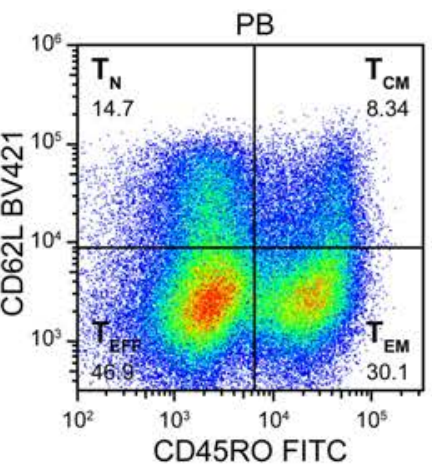

iii

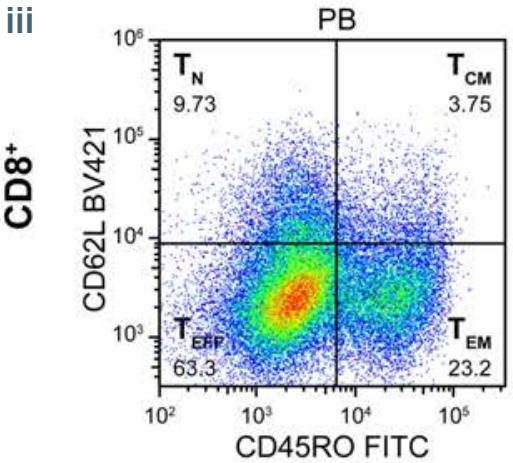

CTRL
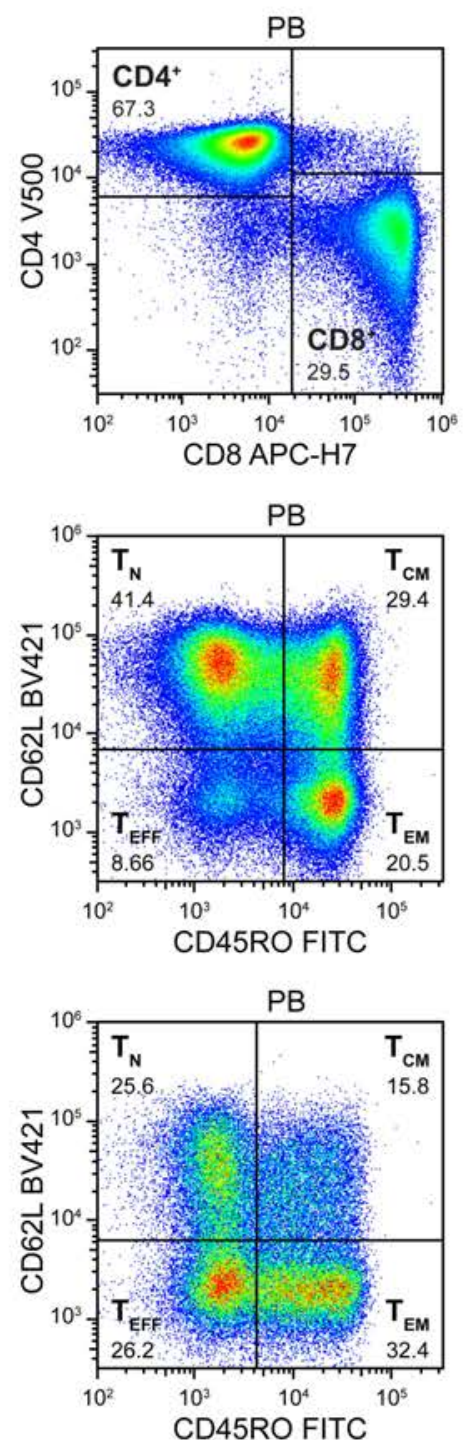

C

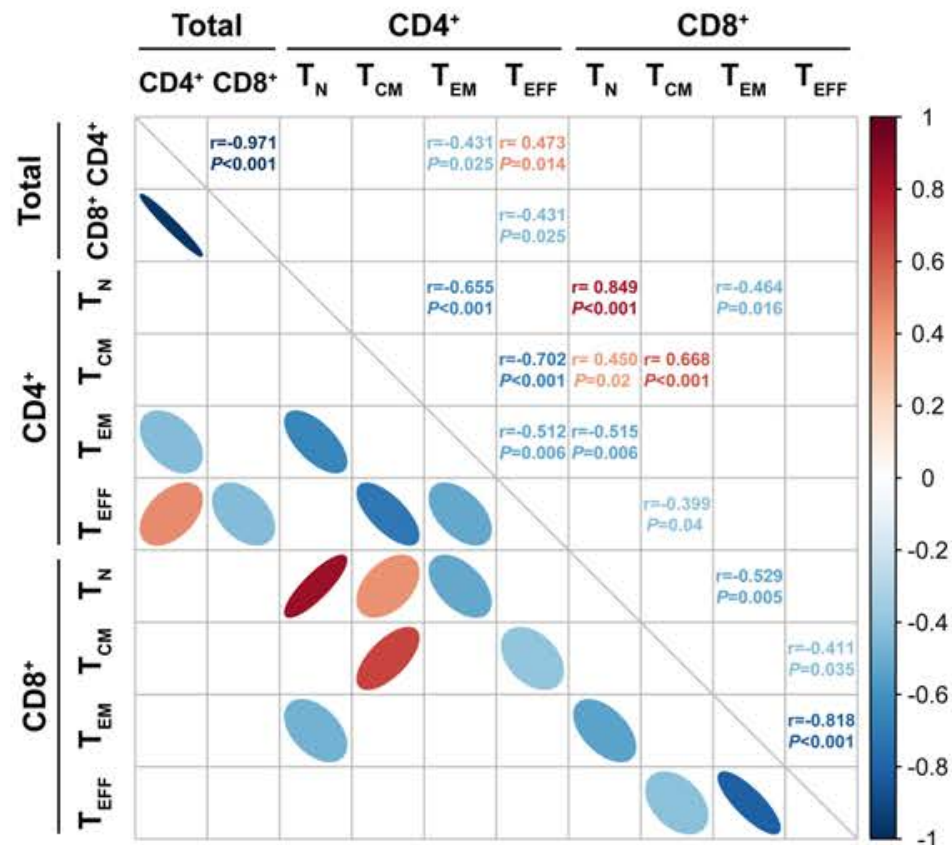

$=\mathrm{MF}=\mathrm{CTRL}$

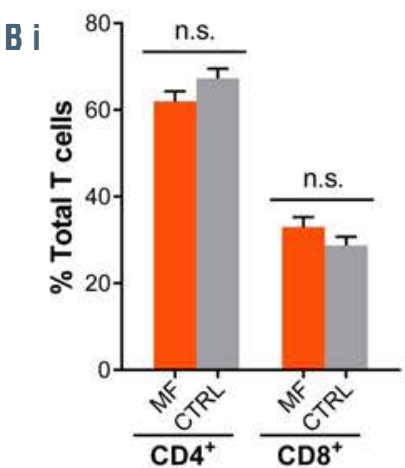

ii

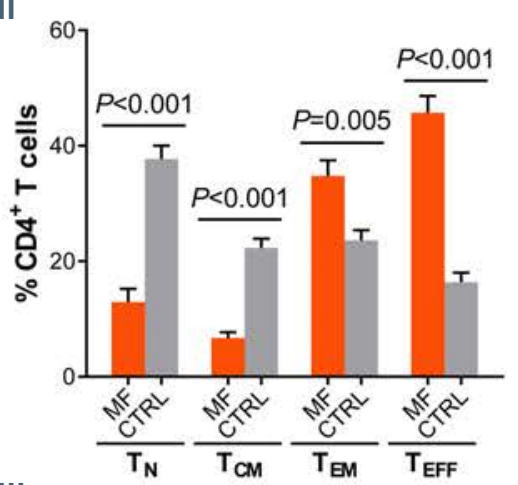

iii

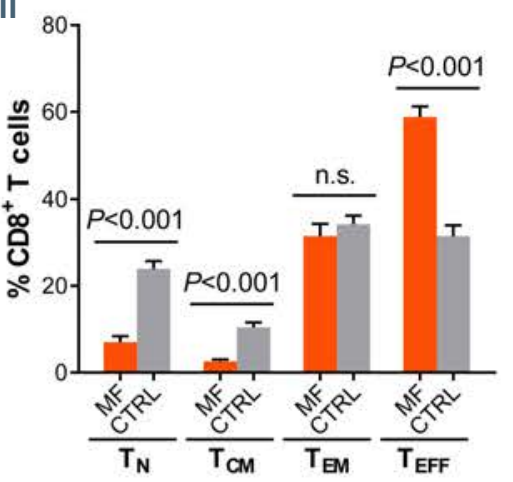

Figure 2. Distribution of T-cell subsets in myelofibrosis. T-cell subsets from patients with myelofibrosis (MF; $n=41$ ) and healthy controls (CTRL; $n=28$ ) were analyzed by flow cytometry. (A) Representative dot plots from a single MF patient and CTRL are depicted. Minimal difference was observed in $\mathrm{CD}^{+}$and $\mathrm{CD}^{+}$ differentiation subsets within the total T cells (i). In contrast, a significant shift in distribution towards effector populations was seen among activation subsets within $\mathrm{CD}^{+}$(ii) and $\mathrm{CD} 8^{+}$(iii) T-cell populations. Whereas the numbers of naïve $\left(T_{*}\right)$ and central memory $T$ cells $\left(T_{c u}\right)$ were reduced, effector memory $(T)$ and effector $T$ cells $\left(T_{\mathrm{EF}}\right)$ were significantly increased, apart from $C D 8^{+} T_{B u}$ cells. (B) Quantification of differentiation subsets (i), and $\mathrm{CD}^{+}$(ii) and $\mathrm{CD}^{+}$(iii) activation subsets from MF patients (red) and CTRL (gray). (C) Correlation matrix showing significant colorcoded relationships (lower half), Pearson coefficients $(r)$, and $P$-values (upper half) between each pair of Tcell subsets. Numbers in dot plots denote percent of cells per gate. Bars represent means with standard deviation. The Student $t$-test was used to compare the two groups. The $P$-values in the correlation matrix were adjusted by the Benjamini-Hochberg method. $P<0.05$ was considered statistically significant. $\mathrm{PB}$, peripheral blood; n.s., not significant. 
time (Figure 3). As shown in a representative patient after 2 years of treatment (Figure 3Ai) and in all patients over the mean treatment period (Figure $\mathrm{Bi}$ ), the percent of $\mathrm{CD} 4^{+}$cells decreased, whereas the percent of $\mathrm{CD}^{+}$cells significantly increased (mean differences $-5.1 \%$ vs. $13.9 \%, P=0.042$ and $P=0.025$, respectively). Within the $\mathrm{CD}^{+}$cell subsets (Figure 3 Aii and Bii), we observed a 2 -fold increase in the percent of $T_{N}$ and $T_{c n}$ cells (mean fold changes 2.05 and 2.26, respectively; $P<0.001$ for both), and 1.2- fold $(P=0.01)$ and 1.48 -fold $(P<0.001)$ decreases in the percent of $T_{\mathrm{E}}$ and $T_{\mathrm{EF}}$ cells, respectively, during ruxolitinib treatment. Comparable effects were observed within the $\mathrm{CD} 8^{+}$cell subsets (Figure 3Aiii and Biii): the percent of $T_{N}$ and $T_{M n}$ cells increased by 2.22-fold and 1.64-fold ( $P=0.002$ and $P=0.03$, respectively), the percent of $\mathrm{CD}^{+} \mathrm{T}_{\mathrm{HF}}$ cells decreased by 1.21-fold $(P=0.001)$, whereas the percent of $\mathrm{CD}^{+} \mathrm{T}_{\mathrm{M}}$ cells remained unaffected by ruxolitinib treatment.
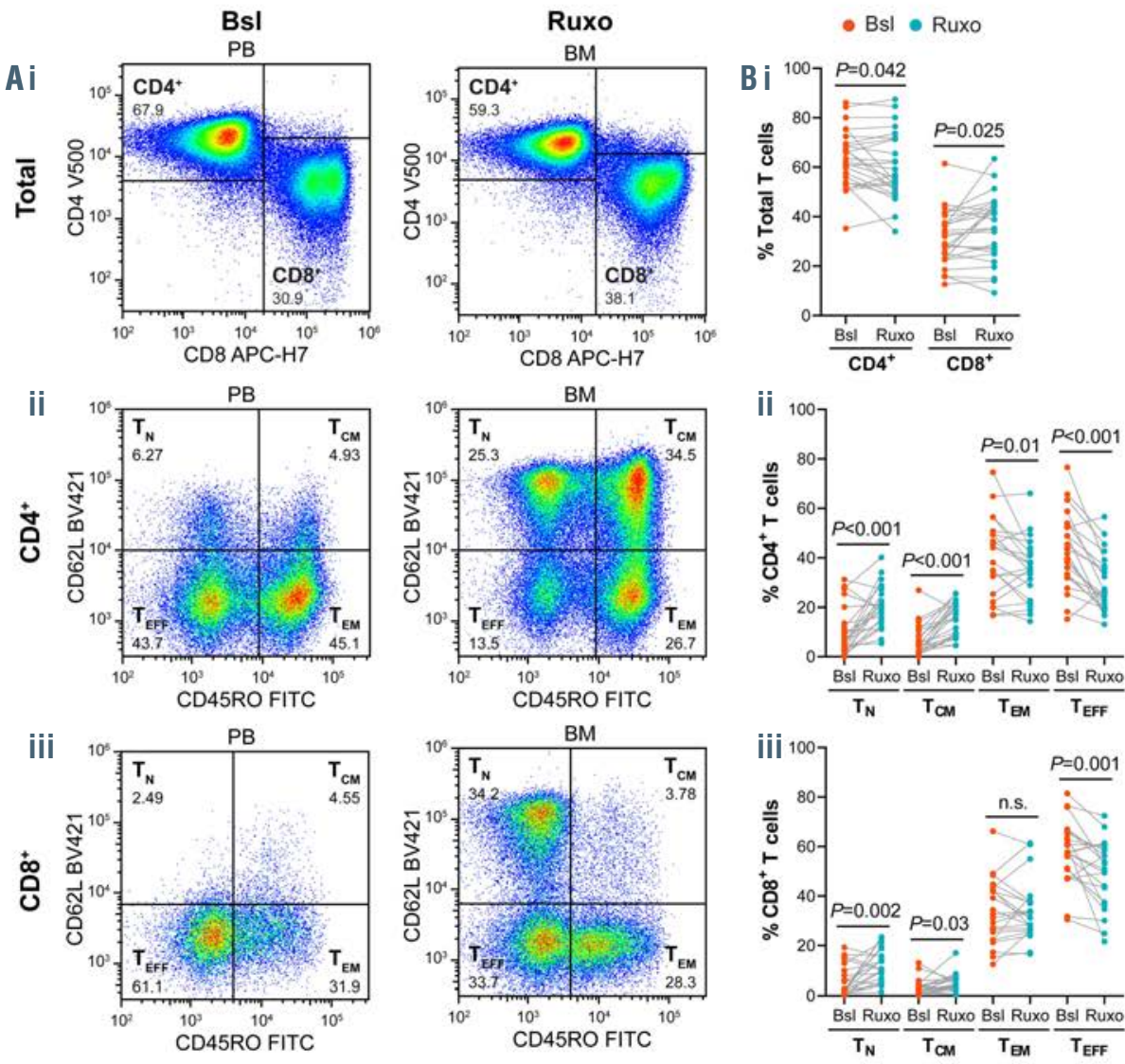

C i
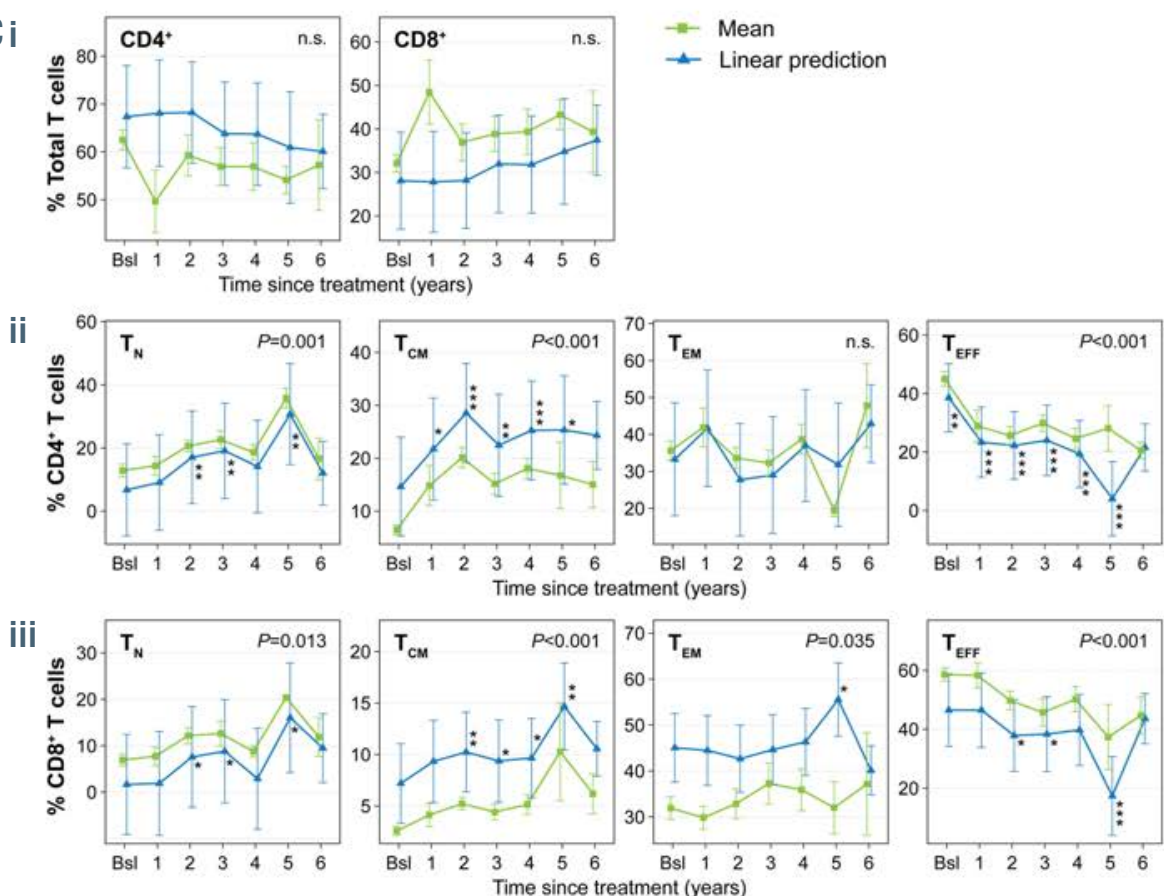

Figure 3. Effects of ruxolitinib treatment on T-cell subsets of patients with myelofibrosis. T-cell subsets from patients with myelofibrosis (MF) $(n=25)$ were analyzed before and over the course of treatment with ruxolitinib. (A) Representative dot plots from a MF patient at treatment baseline (Bsl) and after 2 years of treatment (Ruxo). Naïve ( $\left.T_{n}\right)$ and central memory $T$ cells $\left(T_{c u}\right)$ were increased, whereas effector memory $\left(T_{\mathrm{En}}\right)$ and effector $T$ cells $\left(\mathrm{T}_{\mathrm{EFF}}\right)$ were reduced. Only $\mathrm{CD} 4^{+} \mathrm{T}_{\mathrm{EM}}$ cells did not change significantly with treatment as compared to baseline. (B) Quantification of CD4/CD8 differentiation subsets (i) and activation subsets (ii-iii) at treatment baseline (red) and during the overall follow-up period (light blue). Follow-up values were calculated as a mean of all the analyzed timepoints over the course of treatment for each patient. (C) Longitudinal analysis of T-cell subsets over 6 years of ruxolitinib treatment. The figures shows the mean percentages (green) and linear predictions (dark blue) based on linear mixed-effects model with repeated measures in differentiation (i) and activation subsets (ii-iii). Numbers in dot plots denote the percent of cells per gate. A paired $t$-test was used to compare the two groups. In the longitudinal plots error bars denote the standard error, $P$-values represent the statistical significance of change from baseline over time, and asterisks indicate the significance of change in each year of treatment. Linear mixed models were corrected for spleen size, grade of bone marrow fibrosis, and $J A K 2^{\mathrm{V} 617 \mathrm{~F}}$ allele burden. The $P$ values were computed using the KenwardRoger adjusted F-test. $P$ values $<0.05$ were considered statistically significant. $* P<0.05$; $* * P<0.01 ; \quad * * * P<0.001$. PB: peripheral blood; BM: bone marrow; n.s.: not significant. 
a PD1 PE-labeled T cells 뻬 Other T cells
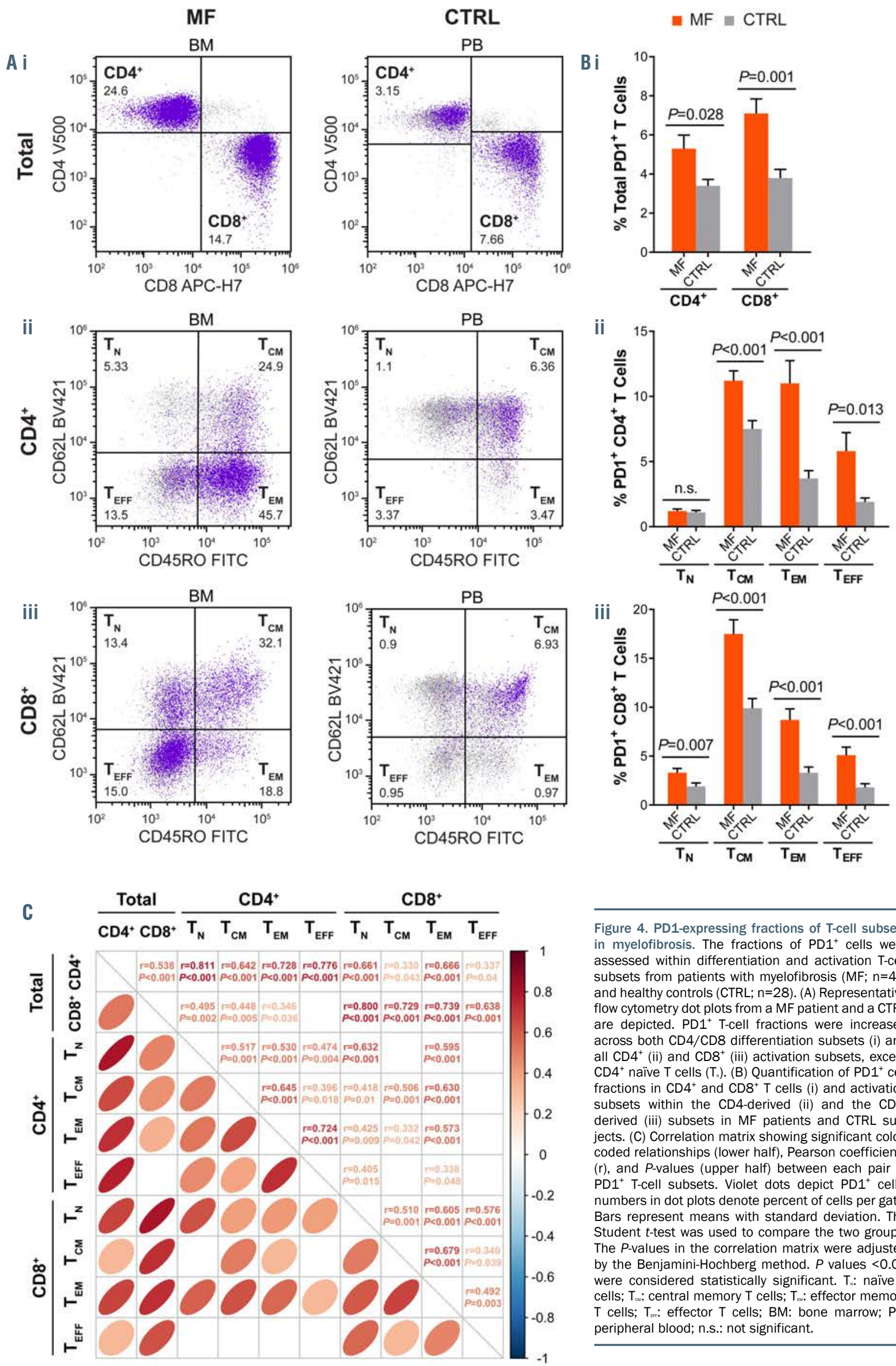

Figure 4. PD1-expressing fractions of T-cell subsets in myelofibrosis. The fractions of $\mathrm{PD}^{+}$cells were assessed within differentiation and activation T-cell subsets from patients with myelofibrosis (MF; $n=41$ ) and healthy controls (CTRL; $n=28$ ). (A) Representative flow cytometry dot plots from a MF patient and a CTRL are depicted. $\mathrm{PD}^{+} \mathrm{T}$-cell fractions were increased across both CD4/CD8 differentiation subsets (i) and all $\mathrm{CD}^{+}$(ii) and $\mathrm{CD} 8^{+}$(iii) activation subsets, except $\mathrm{CD}^{+}$naïve $\mathrm{T}$ cells $\left(\mathrm{T}_{*}\right)$. (B) Quantification of $\mathrm{PD} 1^{+}$cell fractions in $\mathrm{CD}^{+}$and $\mathrm{CD} 8^{+} \mathrm{T}$ cells (i) and activation subsets within the CD4-derived (ii) and the CD8derived (iii) subsets in MF patients and CTRL subjects. (C) Correlation matrix showing significant colorcoded relationships (lower half), Pearson coefficients $(r)$, and $P$-values (upper half) between each pair of PD1 ${ }^{+}$T-cell subsets. Violet dots depict PD $1^{+}$cells; numbers in dot plots denote percent of cells per gate. Bars represent means with standard deviation. The Student $t$-test was used to compare the two groups. The $P$-values in the correlation matrix were adjusted by the Benjamini-Hochberg method. $P$ values $<0.05$ were considered statistically significant. $T_{w}$ : naïve $T$ cells; $T_{\text {cn }}$ : central memory $T$ cells; $T_{\text {en: }}$ : effector memory T cells; $T_{\mathrm{err}}$ : effector T cells; BM: bone marrow; PB, peripheral blood; n.s.: not significant. 
To investigate dynamic changes in T-cell surface marker expression over the course of ruxolitinib treatment, consecutive cell surface marker profiles were analyzed using mixed linear models with repeated measures. To account for the progressive nature of $\mathrm{MF}$, our final model also included spleen size, grade of $\mathrm{BM}$ fibrosis, and $J A K 2^{\mathrm{V} 617 \mathrm{~F}}$ allele burden (Online Supplementary Tables S3 and S4). After correcting for these variables, we observed no significant change in $\mathrm{CD}^{+}$and $\mathrm{CD} 8^{+} \mathrm{T}$ cells over the course of ruxolitinib treatment (Figure $3 \mathrm{Ci}$ ), suggesting that the increase in cytotoxic $T$ cells that we observed over the whole treatment period is a result of disease progression rather than an effect of JAK inhibition. In contrast, after correction we still observed time-dependent shifts from effector to resting Tcell subsets (Figure 3Cii-iii), confirming our hypothesis that long-term ruxolitinib treatment mitigates $\mathrm{T}$-cell overactivation. Whereas significant $T_{N}$ and $T_{c}$ cell increases were observed in the second, third and fifth years of therapy, in both $\mathrm{CD} 4^{+}$and $\mathrm{CD} 8^{+}$ subsets $T_{\text {护 }}$ cells consistently decreased over the same period. Similar changes were also observed in the fourth year of treatment, although they reached statistical significance only in the $\mathrm{T}_{\mathrm{Cm}}$ and $\mathrm{CD}^{+} \mathrm{T}_{\mathrm{Fm}}$ subsets. Remarkably, both $\mathrm{T}_{\mathrm{m}}$ subsets showed no significant change during treatment, except for the $\mathrm{CD}^{+}$subset during the fifth year of therapy, suggesting that long-term ruxolitinib treatment prevents terminal activation of $T$ cells in MF, but has little effect on the effector memory arm of T-cell activation.

To determine whether baseline distributions of T-cell differentiation and activation subsets affect the overall survival, datasets were further analyzed using the Kaplan-Meier method and no significant differences were found (Online Supplementary Figure S1). In summary, these data suggest that ruxolitinib treatment shifts the activation state of $\mathrm{T}$-cell subsets from terminal effector towards resting phenotype in a timedependent manner.

\section{PD1-expressing fractions within the T-cell subsets of myelofibrosis patients}

Because it was recently reported that MF myeloid cells express high levels of PDL1, ${ }^{19}$ we sought to evaluate PD1expressing fractions within T-cell subsets of MF patients. The proportion of cells co-expressing PD1 in $\mathrm{CD}^{+}$and $\mathrm{CD} 8^{+} \mathrm{T}$ cells of MF patients ( $\mathrm{n}=35)$ was higher by $55.9 \% \quad(P=0.028)$ and $86.8 \%(P=0.001)$, respectively, compared to $\mathrm{T}$ cells of healthy controls $(n=28)$ (Figure $4 \mathrm{Ai}$ and $\mathrm{Bi})$. Specifically, PD1+ fractions were increased within both $\mathrm{CD}^{+}$and $\mathrm{CD}^{+} \mathrm{T}_{\mathrm{CM}}, \mathrm{T}_{\mathrm{FH}}$, and $\mathrm{T}_{\mathrm{m}}$ cells (mean fold-changes, 1.49, 2.97, and 3.05 in $\mathrm{CD}^{+}$cells; 1.77, 2.64 , and 2.83 in $\mathrm{CD}^{+}$cells, respectively; $P=0.013$ in $\mathrm{CD}^{+} \mathrm{T}_{\mathrm{H}}$, $P<0.001$ in the rest), and within $\mathrm{CD}^{+} \mathrm{T}_{N}$ cells (mean foldchange, 1.74; $P=0.007$ ) (Figure 4Aii-iii and Bii-iii). Importantly, most $\mathrm{PD} 1^{+}$fractions correlated positively between one another (Figure 4C) while no significant correlation was observed with any of the $\mathrm{T}$-cell subsets, suggesting that $\mathrm{PD} 1^{+}$cells are prevalent among MF T cells irrespective of their differentiation or activation state. In addition, we analyzed how ruxolitinib affects PD1 ${ }^{+}$ CD4/CD8 and activation subsets over the whole follow-up period and in each year of treatment, corrected for the parameters of disease progression (spleen size, BM fibrosis grade, and JAK2 $2^{\mathrm{V} 617 \mathrm{~F}}$ allele burden). Overall, no significant differences were observed in $\mathrm{PD} 1^{+}$fractions over the course of ruxolitinib treatment (Online Supplementary Figure S2).

\section{Association between T-cell subsets, PD1 ${ }^{+}$fractions, and disease progression}

Because MF is a progressive myeloproliferative neoplasm, ${ }^{28}$ and $\mathrm{T}$ cells are known to interact with clonal neoplastic cells, ${ }^{29}$ we analyzed the correlation between $\mathrm{T}$-cell subsets and $\mathrm{PB}$ cell counts of untreated MF patients $(n=41)$. We found that the number of $\mathrm{CD}^{+}$ cells correlated positively with monocyte counts and negatively with platelet counts ( $\mathrm{r}=0.317$ and $\mathrm{r}=-.335 ; P=0.043$ and $P=0.032$, respectively); however, $\mathrm{CD} 4^{+}$cell subsets had a negative and positive correlation with monocyte and platelet counts $(\mathrm{r}=-0.371$ and $\mathrm{r}=0.375$, respectively; $P=0.017$ and $P=0.016$, respectively) (Figure $5 \mathrm{Ai}$. Given that both monocytosis and thrombocytopenia are associated with disease progression, ${ }^{30}$ it is likely that $\mathrm{CD}^{+}$cells expand with disease propagation in untreated MF patients. Conversely, increased PD1 levels of both $\mathrm{CD}^{+}$and $\mathrm{CD}^{+}$cells correlated with total leukocyte counts $(\mathrm{r}=0.628$ and $\mathrm{r}=0.547$, respectively; $P<0.001$ for both) and palpable spleen size $(\mathrm{r}=0.435$ and $\mathrm{r}=0.465 ; P=0.005$ and $P=0.002$, respectively Figure 5Aii), suggesting that the increase in $\mathrm{PD} 1^{+} \mathrm{T}$-cell fractions, typically associated with T-cell exhaustion, correlates with disease progression, regardless of $\mathrm{PD} 1$ distribution across those $\mathrm{T}$-cell subsets.

To investigate the effect of disease progression on subset levels at baseline and following treatment with ruxolitinib, we stratified patients based on spleen size, BM fibrosis grade, and $J A K 2^{\mathrm{V} 617 \mathrm{~F}}$ allele burden, and compared their total, $\mathrm{T}_{\mathrm{M}}$ and $\mathrm{PD} 1^{+}$subsets, using healthy controls as a reference (Figure 5B). Although we found a $29.5 \%$ larger $\mathrm{CD}^{+} \mathrm{T}$-cell population in MF patients with a palpable spleen larges than $20 \mathrm{~cm}$ at treatment baseline $(\mathrm{n}=11)$, this effect did not reach statistical significance $(P=0.087)$. Interestingly, however, we also found $17.1 \%$ fewer $\mathrm{CD} 4^{+}$cells in this group of patients than in the control group $(P=0.027)$. Moreover, MF patients with advanced-stage disease prior to treatment did not exhibit the significant repolarization of CD4/CD8 populations over time shown by patients with early-stage disease, further indicating that CD8 predominance is not a ruxolitinib effect but a result of disease progression. MF patients with splenomegaly greater than $20 \mathrm{~cm}$ had 1.4-fold larger baseline $\mathrm{CD} 4^{+} \mathrm{T}_{\mathrm{m}}$ subsets and $\mathrm{CD} 4^{+} \mathrm{PD} 1^{+}$fractions $(P=0.045$ and $P=0.029$, respectively) compared to patients with smaller spleens. Of note, both $\mathrm{PD} 1^{+} \mathrm{CD}^{+}$and $\mathrm{CD} 8^{+}$subsets of these patients were significantly higher than normal ( $P=0.003$ and $P=0.013$, respectively), similar to patients with MF-3 grade fibrosis $(P=0.042$ and $P<0.001$, respectively) and patients with mutant $J A K 2$ allele burden above $50 \%\left(P=0.038\right.$ and $P=0.002$, respectively). Overall, the $\mathrm{CD}^{+} \mathrm{T}_{\mathrm{H}}$ subset showed little difference based on the analyzed parameters of disease progression, supporting the idea that $\mathrm{CD} 8^{+}$resting cells in MF rapidly transit to T-effectors as they become activated. Remarkably, patients with high mutant JAK2 allele burden had significantly lower numbers of $\mathrm{CD}^{+} \mathrm{T}_{\mathrm{IM}}(P=0.022)$.

\section{Association between T-cell subsets, $\mathrm{PD}^{+}$fractions, and clinical response to ruxolitinib}

Because a reduction in spleen size is typically associated with a good response to ruxolitinib and favorable treatment outcome, ${ }^{24,26,27}$ we tested the association between pretreatment $\mathrm{T}$-cell subsets and spleen size 6 months into therapy. We found that complete resolution of palpable splenomegaly was associated with an increased percent of $\mathrm{CD}^{+}$cells and a decreased percent of $\mathrm{CD} 8^{+}$cells (mean differences $14.4 \%$ and $-23 \% ; P=0.038$ and $P=0.049$, respectively) (Figure 6A). Furthermore, complete resolution of palpable splenomegaly was associated with a low percent of $\mathrm{PD} 1^{+}$fractions in both $\mathrm{CD}^{+}$and $\mathrm{CD}^{+}$cell subsets (mean differences, $-30.7 \%$ and $-31.7 \% ; P=0.012$ and $P=0.036$, respectively) (Figure $6 \mathrm{~B}$ ), suggesting that MF patients with low levels of exhausted $\left(\mathrm{PD} 1^{+}\right) \mathrm{T}$ cells likely respond favorably to ruxolitinib treatment.

\section{Effect of PD1 ${ }^{+}$T-cell fractions on survival rates of myelofibrosis patients}

Data from $41 \mathrm{MF}$ patients were further analyzed using the Kaplan-Meier method (34 patients [82.9\%] had died) to determine whether the distribution of $\mathrm{PD}^{+} \mathrm{T}$-cell fractions affects 
A i

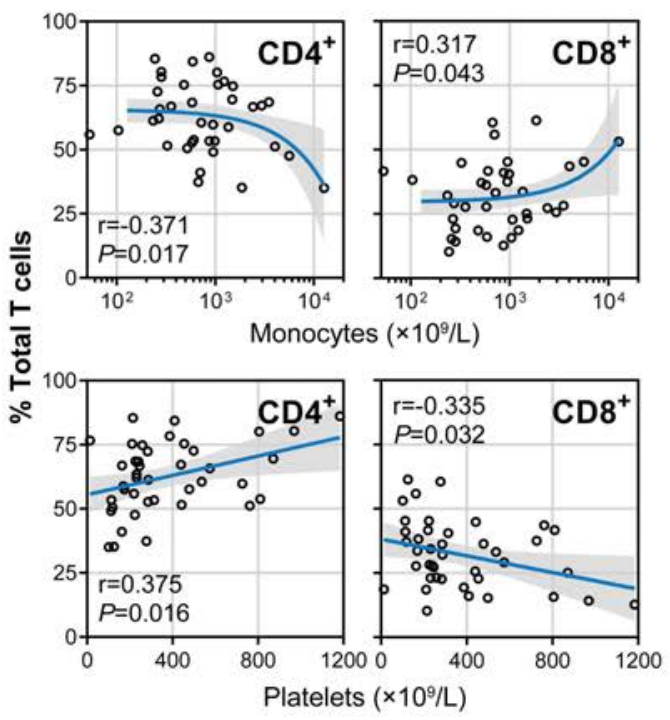

ii

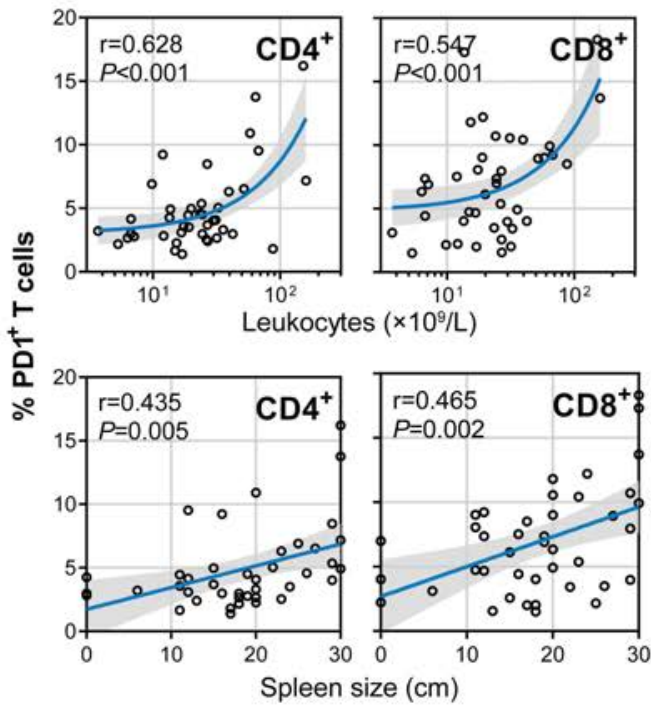

B

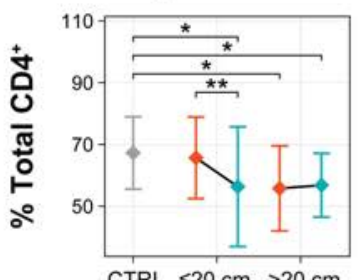

CTRL $\leq 20 \mathrm{~cm}>20 \mathrm{~cm}$

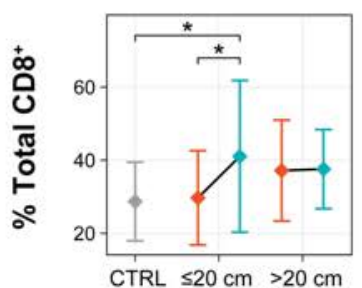

CTRL $\leqslant 20 \mathrm{~cm}>20 \mathrm{~cm}$
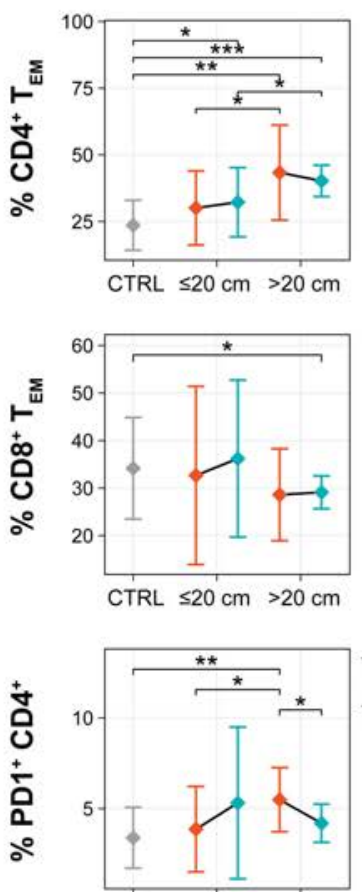

CTRL $\leq 20 \mathrm{~cm}>20 \mathrm{~cm}$

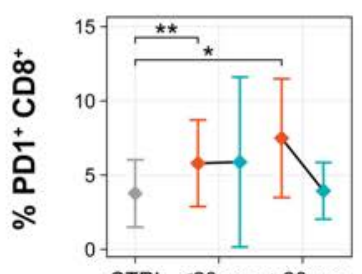

CTRL $\leq 20 \mathrm{~cm}>20 \mathrm{~cm}$

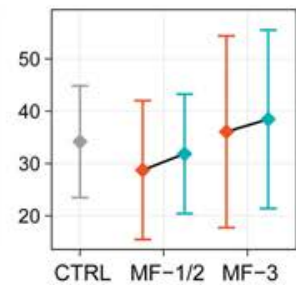

CTRL \& BsI \& Ruxo

BM fibrosis grade

$\%$ JAK2 ${ }^{\mathrm{V} 617 \mathrm{~F}}$

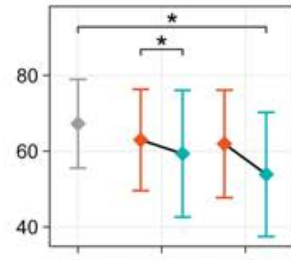

CTRL MF-1/2 MF-3
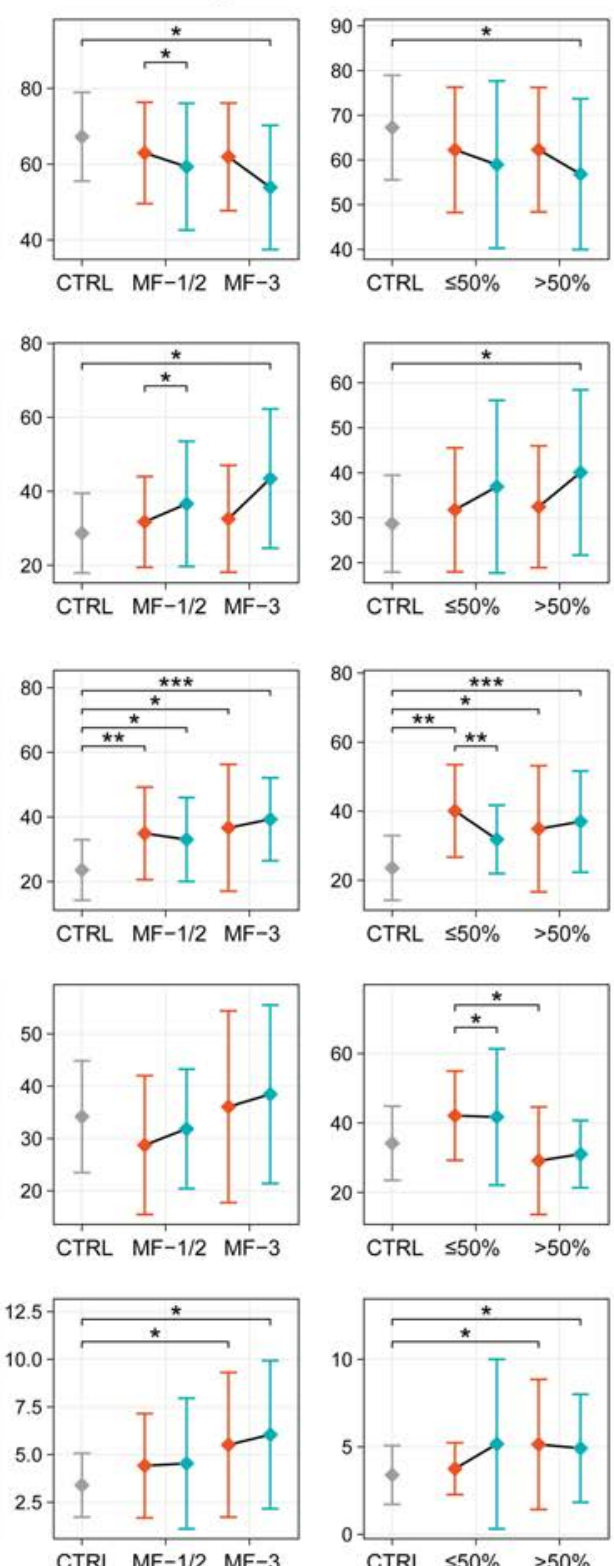

CTRL $\leq 50 \% \quad>50 \%$

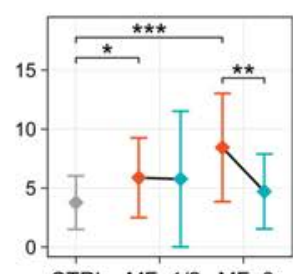

CTRL MF-1/2 MF-3

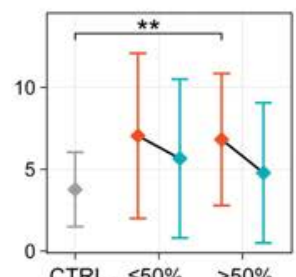

Figure 5. Association between myelofibrosis T-cell subsets and PD1 ${ }^{+}$fractions with disease progression. Correlations between T-cell subsets and PD $1^{+}$fractions with peripheral blood cell counts and spleen size were assessed at treatment baseline. Pre- and on-treatment T cells were also compared based on selected parameters of disease progression/reduction. (A) Correlation of $\mathrm{CD} 4^{+}$and $\mathrm{CD} 8^{+} \mathrm{T}$-cell subsets with absolute monocyte and platelet counts (i), and correlation of PD $1^{+} / \mathrm{CD} 4^{+}$ and $\mathrm{PD} 1^{+} / \mathrm{CD}^{+} \mathrm{T}$-cell percent with absolute leukocyte counts and spleen size (ii). (B) Comparison of CD4 ${ }^{+}$and $\mathrm{CD} 8^{+}$total $\mathrm{T}$ cells, effector memory $\mathrm{T}$ cell ( $\mathrm{T}_{\mathrm{Eu}}$ ) subsets, and PD1 ${ }^{+}$fractions based on the baseline spleen size, bone marrow (BM) fibrosis grade, and JAK2 ${ }^{\mathrm{V} 617 \mathrm{~F}}$ allele burden. Data are shown for healthy control (CTRL; gray), baseline (Bsl; red), and ruxolitinib-treated (Ruxo; light blue) groups. Follow-up values were calculated as a mean of all the analyzed time- points over the course of treatment. Spleen size was defined by physical examination (the measured distance of palpable spleen edge from the left costal margin in the left midclavicular line) with the cutoff value of $20 \mathrm{~cm}$. BM fibrosis grade was assessed in accordance with European consensus criteria. Mutant JAK2 allele burden was quantified using quantitative polymerase chain reaction analysis and dichotomized using the cutoff value of $50 \%$. Regression lines are shown in blue; gray shaded areas denote $95 \%$ confidence intervals. Dots with error bars represent means with standard deviation. The Pearson coefficient was used to determine the degree of correlation. A Welch or paired $t$-test was used to compare the two groups. $P$ values $<0.05$ were considered statistically significant. $* P<0.05 ; * * P<0.01 ; * * * P<0.001$. n.s.: not significant. 
A
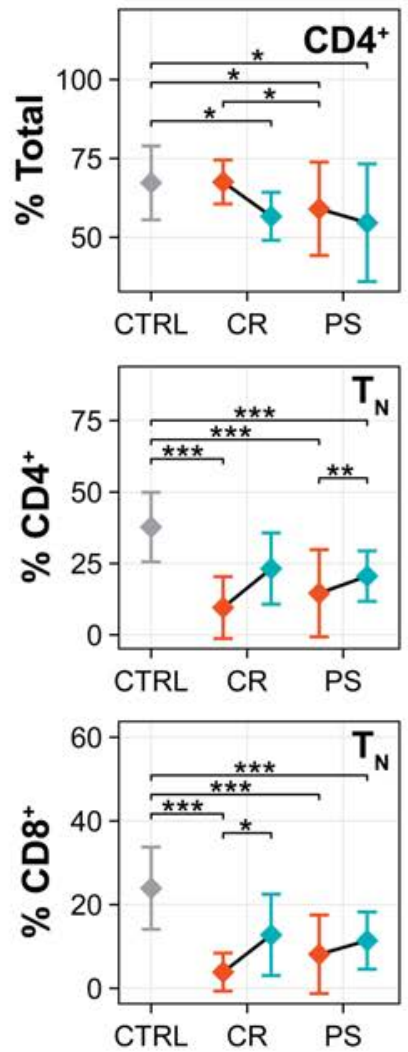

B

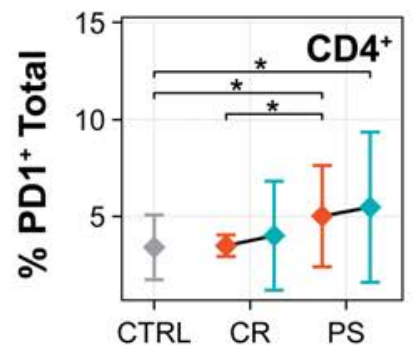

CTRL CR PS
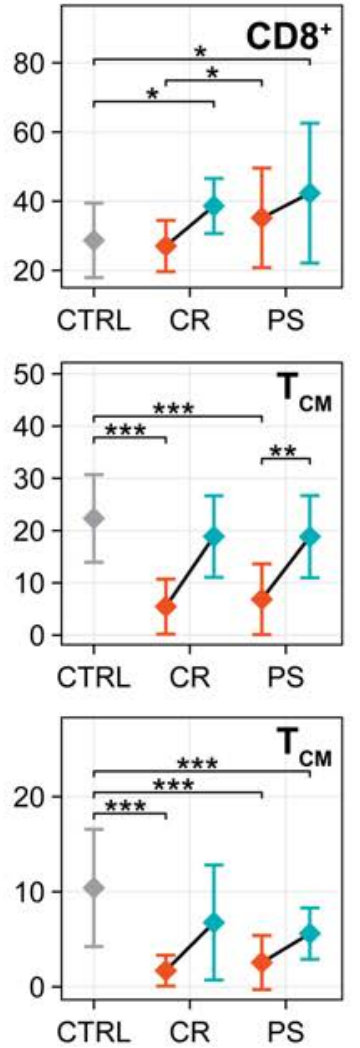

$\$$ CTRL

\& Bsl

$\uparrow$ Ruxo
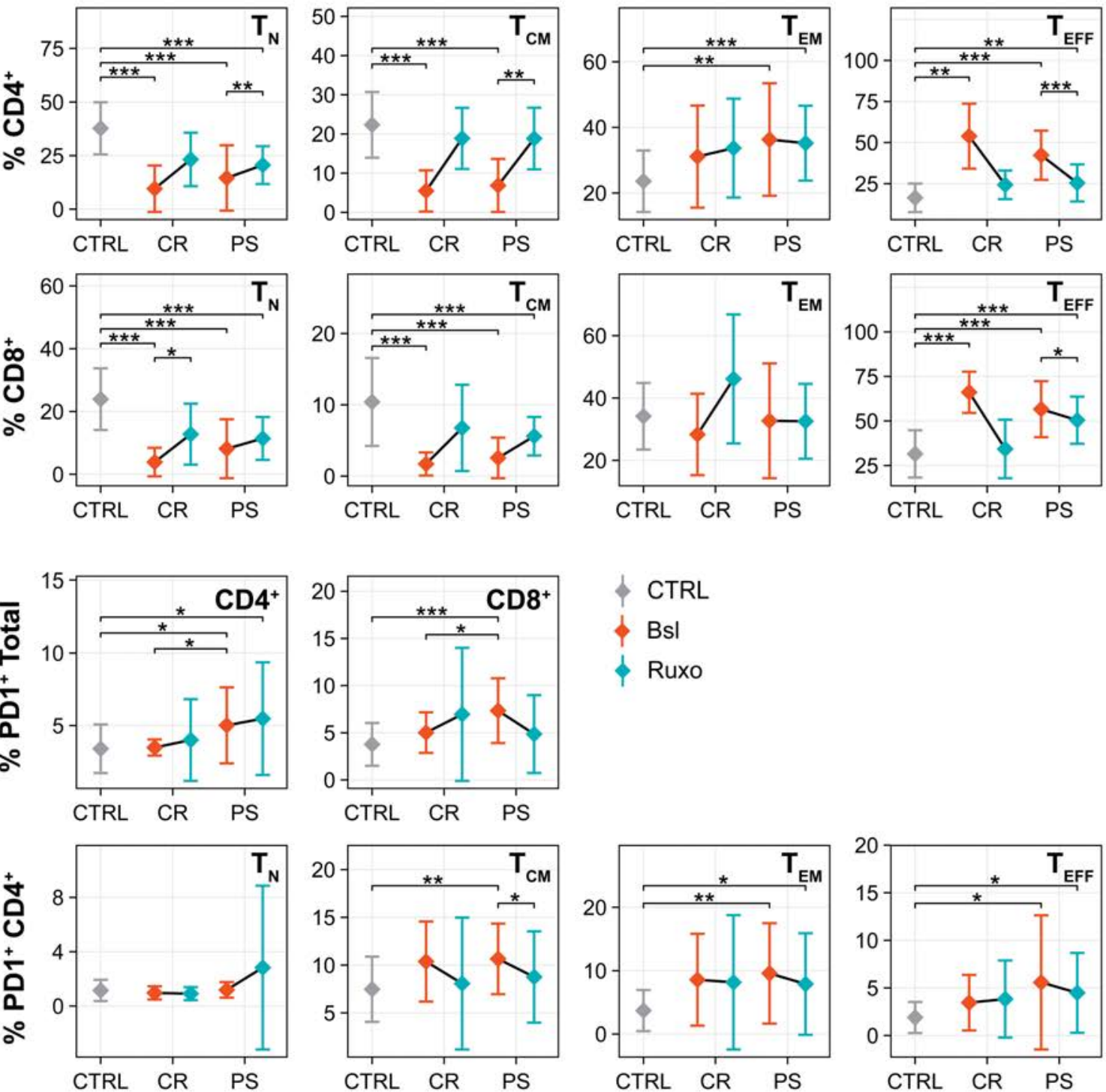

$$
\begin{aligned}
& \$ \text { CTRL } \\
& \$ \text { BsI } \\
& \$ \text { Ruxo }
\end{aligned}
$$

CTRL CR PS
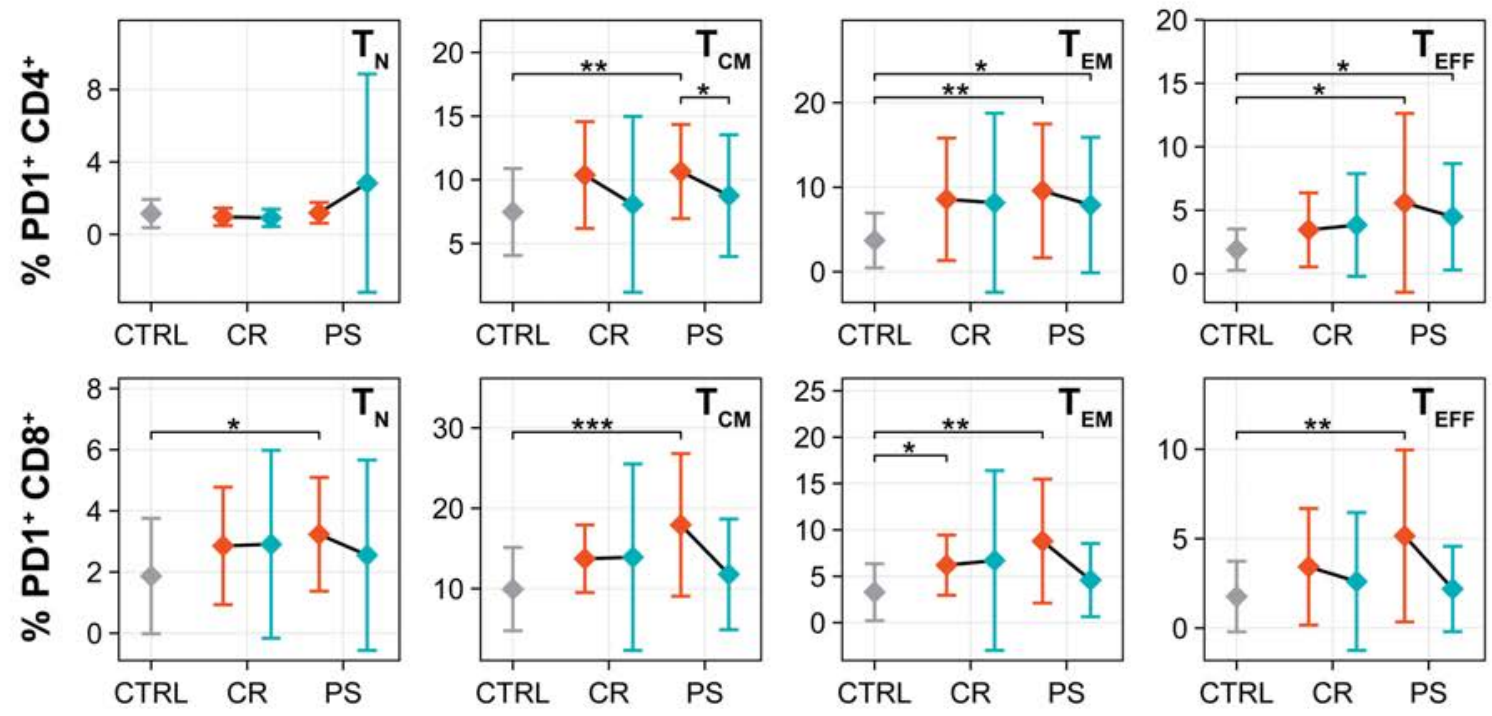

Figure 6. Association of myelofibrosis T-cell subsets and PD1 ${ }^{+}$fractions with clinical response to ruxolitinib treatment. (A, B) Comparison of T-cell subset (A) and $\mathrm{PD}^{+}$fraction (B) distribution based on spleen response of patients with myelofibrosis 6 months into ruxolitinib treatment. Spleen response was classified as either complete resolution (CR; $n=9$ ) or persistent splenomegaly (PS; $n=29$ ). CR was defined as no palpable splenomegaly after 6 months of treatment in patients with $\geq 5$ $\mathrm{cm}$ of palpable spleen at treatment baseline. $P$ values $<0.05$ were considered statistically significant. $* P<0.05 ; * \star P<0.01 ; * * \star P<0.001$. 
the outcome of ruxolitinib treatment. Using the log-rank test, we found that an increase of $\mathrm{CD} 4^{+} / \mathrm{PD} 1^{+}$cells above $3.31 \%$ or of $\mathrm{CD}^{+} / \mathrm{PD} 1^{+}$cells above $6.12 \%$ was associated with a poor overall survival ( $P=0.014$ and $P=0.003$, respectively) (Figure 7Ai). However, significant differences in survival were maintained only across $\mathrm{CD}^{+}$, and not $\mathrm{CD}^{+}, \mathrm{T}_{\mathrm{N}}, \mathrm{T}_{\mathrm{cm}}, \mathrm{T}_{\mathrm{m}}$, and $\mathrm{T}_{\mathrm{EF}}$ cells ( $P=0.002, P=0.013, P=0.009$, and $P=0.007$, respectively) (Figure
7Aii-iii), suggesting that increased $\mathrm{PD} 1^{+} \mathrm{T}$-cell fractions, typically associated with $\mathrm{T}$-cell exhaustion, are a better predictor of outcome within the cytotoxic T-cell subset. A univariate Cox proportional hazard regression analysis of the clinical variables revealed that disease subtype (primary MF vs. secondary MF), BM fibrosis grade (MF-3 vs. MF-1/2), transformation to acute myeloid leukemia, or transfusion dependence were significant
$\mathrm{Ai}$
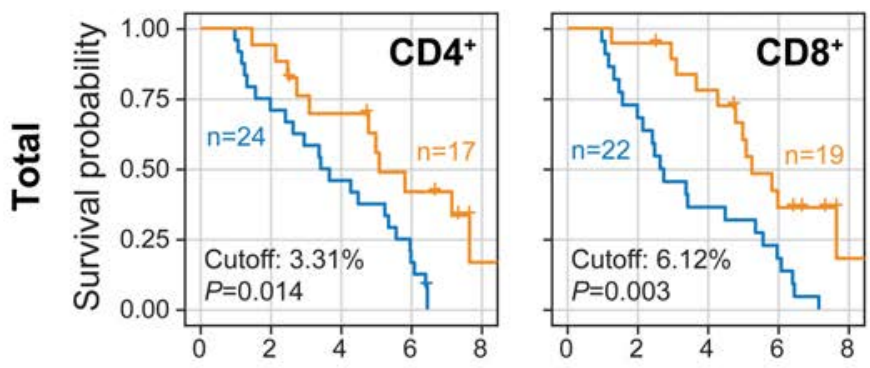

Time since treatment (years)

ii

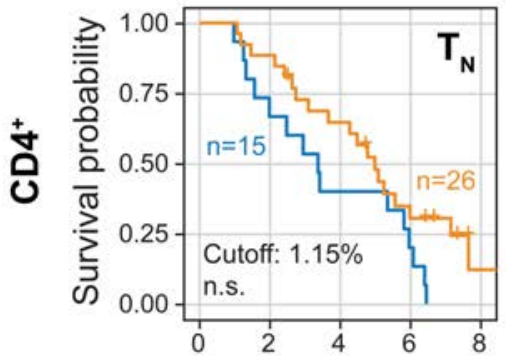

iii

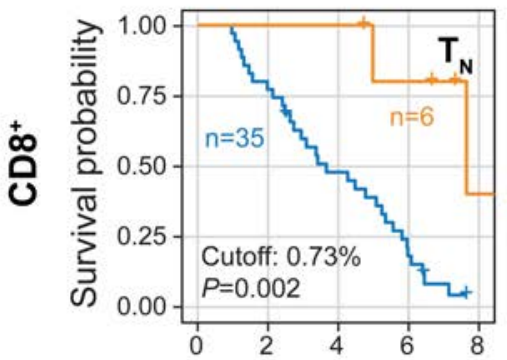

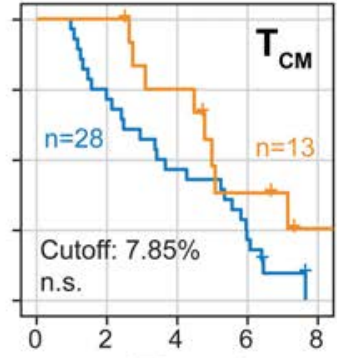

Time since treatment (years)
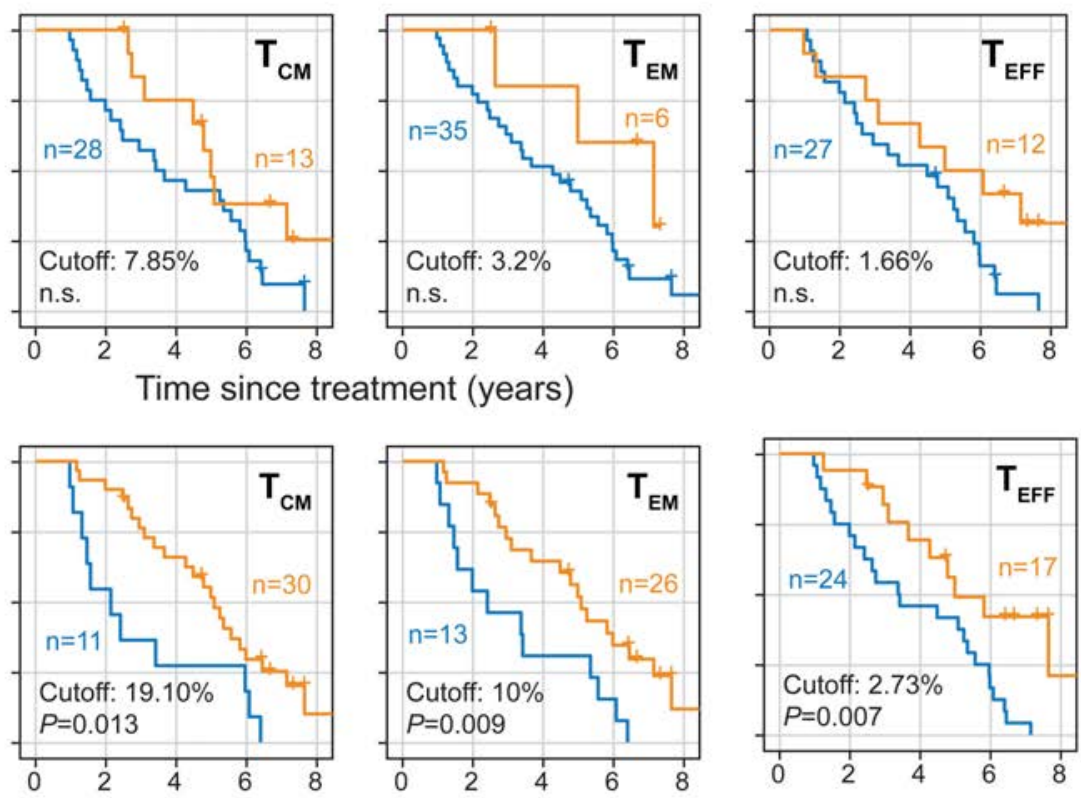

Time since treatment (years)

B

Predictors
$\uparrow \mathrm{PD} 1^{+} \mathrm{CD} 8^{+} \mathrm{T}$-cell \%
Transformation to AML
Transfusion dependence
PMF vs PPV/PET MF
MF-3 vs MF-1/2
$\uparrow \mathrm{PD} 1^{+} \mathrm{CD} 4^{+} \mathrm{T}$-cell \%

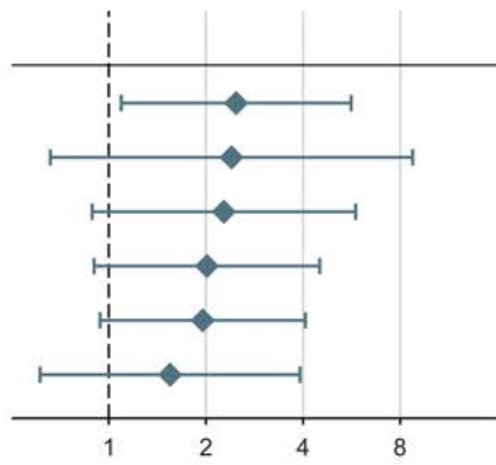

\begin{tabular}{lcc} 
HR & $\mathrm{Cl}$ & $\boldsymbol{P}$ \\
\hline 2.48 & $(1.09-5.64)$ & 0.03 \\
2.40 & $(0.66-8.74)$ & 0.184 \\
2.27 & $(0.89-5.82)$ & 0.087 \\
2.01 & $(0.90-4.50)$ & 0.088 \\
1.95 & $(0.94-4.07)$ & 0.073 \\
1.55 & $(0.61-3.91)$ & 0.355
\end{tabular}

Figure 7. Association of baseline PD1 $1^{+}$T-cell fractions with the clinical outcome of ruxolitinib-treated patients with myelofibrosis. Associations between the survival of patients with myelofibrosis (MF) and percent of PD1 ${ }^{+} \mathrm{T}$ cells in differentiation and activation subsets at the treatment baseline were analyzed using the KaplanMeier method and Cox models. (A) Kaplan-Meier survival analysis based on percent of PD1 ${ }^{+}$cells in CD4/CD8 differentiation subsets (i), and in CD4 ${ }^{+}$(ii) and CD8 ${ }^{+}$ (iii) activation subsets. (B) Results of multivariate analysis of survival using high versus low PD $1^{+} / \mathrm{CD} 4^{+}$and $\mathrm{PD} 1^{+} / \mathrm{CD} 8^{+}$baseline T-cell subsets are shown. Cutoff values for dichotomization of each subset into high and low groups were determined using the maximally selected rank statistic. $P$-values for differences in overall survival were calculated using the log-rank test. Each multivariate model also included disease subtype (primary [PMF] vs. secondary MF [PPV/PET MF]), grade of bone marrow fibrosis (MF-3 vs. MF-1/2), transformation to acute myeloid leukemia (AML), and transfusion dependence as confounding predictors. Rhombi depict the hazard ratio (HR) of each predictor, and lines represent $95 \%$ confidence intervals $(\mathrm{Cl})$. $P$ values $<0.05$ were considered statistically significant. n.s.: not significant. 
confounding factors for overall survival in this cohort of patients. We therefore performed a multivariate analysis of survival after adjusting the model for these four predictors (Figure $7 \mathrm{~B}$ ). This analysis confirmed that PD1-overexpressing fractions of $\mathrm{CD}^{+}$ cells, but not $\mathrm{CD}^{+}$cells, before initiation of ruxolitinib treatment, independently predicted overall survival (hazard ratio: 2.48; $P=0.03$ ). Taken together, our results demonstrated that increased $\mathrm{PD} 1^{+} / \mathrm{CD}^{+} \mathrm{T}$-cell subsets were significantly associated with a high risk of death in ruxolitinib-treated MF patients.

\section{Discussion}

In the current study, we found that T-cell subsets of patients with MF shifted from a quiescent to an activated state and that treatment with ruxolitinib reduced the activation of both helper CD4 and cytotoxic CD8 T cells in a time-dependent manner (Figure 8). The activation pattern of $\mathrm{CD}^{+} \mathrm{T}$ cells was significantly similar to that of polycythemia vera, ${ }^{31}$ including decreased $T_{N}$ and $T_{\mathrm{C} M}$, unaltered $\mathrm{T}_{\mathrm{EM}}$ and increased $\mathrm{T}_{\mathrm{EF}}$ subsets. However, in MF, CD4 ${ }^{+}$ cells were also considerably skewed toward an effector cell phenotype, unlike polycythemia vera. Whereas $\mathrm{CD} 8^{+}$cells are activated by major histocompatibility complex (MHC) type I molecules, priming of $\mathrm{CD}^{+} \mathrm{T}$ cells is restricted to $\mathrm{MHC}$ class II on predominantly monocyte-derived antigen-presenting cells. Because MHC expression is induced by activated JAK $2,{ }^{32}$ our data point toward a predominant role of neoplastic monocytes in aberrant $\mathrm{T}$-cell responses in MF. Furthermore, circulating monocyte-derived dendritic cells from patients with MF were extremely efficient in antigen uptake as compared to dendritic cells from healthy individuals, despite their reduced numbers and function. ${ }^{33}$ In comparison, T cells of acute myeloid leukemia patients at diagnosis are predominantly $\mathrm{CD}^{+}, 34,35$ whereas their activation seems to differ minimally from normal cells in both $\mathrm{CD}^{+}{ }^{+}$and $\mathrm{CD}^{+}$subsets. ${ }^{36,37}$ Overall, the activation status of $\mathrm{T}$-cell subsets in patients with $\mathrm{MF}$ is consistent with an ongoing antineoplastic immune response, characteristic of the "T-cell inflamed" immune signature. ${ }^{38}$

Over the last decade, broad clinical experience has been acquired in treating MF patients with ruxolitinib. Overall, decreased rates of infections and spleen reduction with ruxoli-

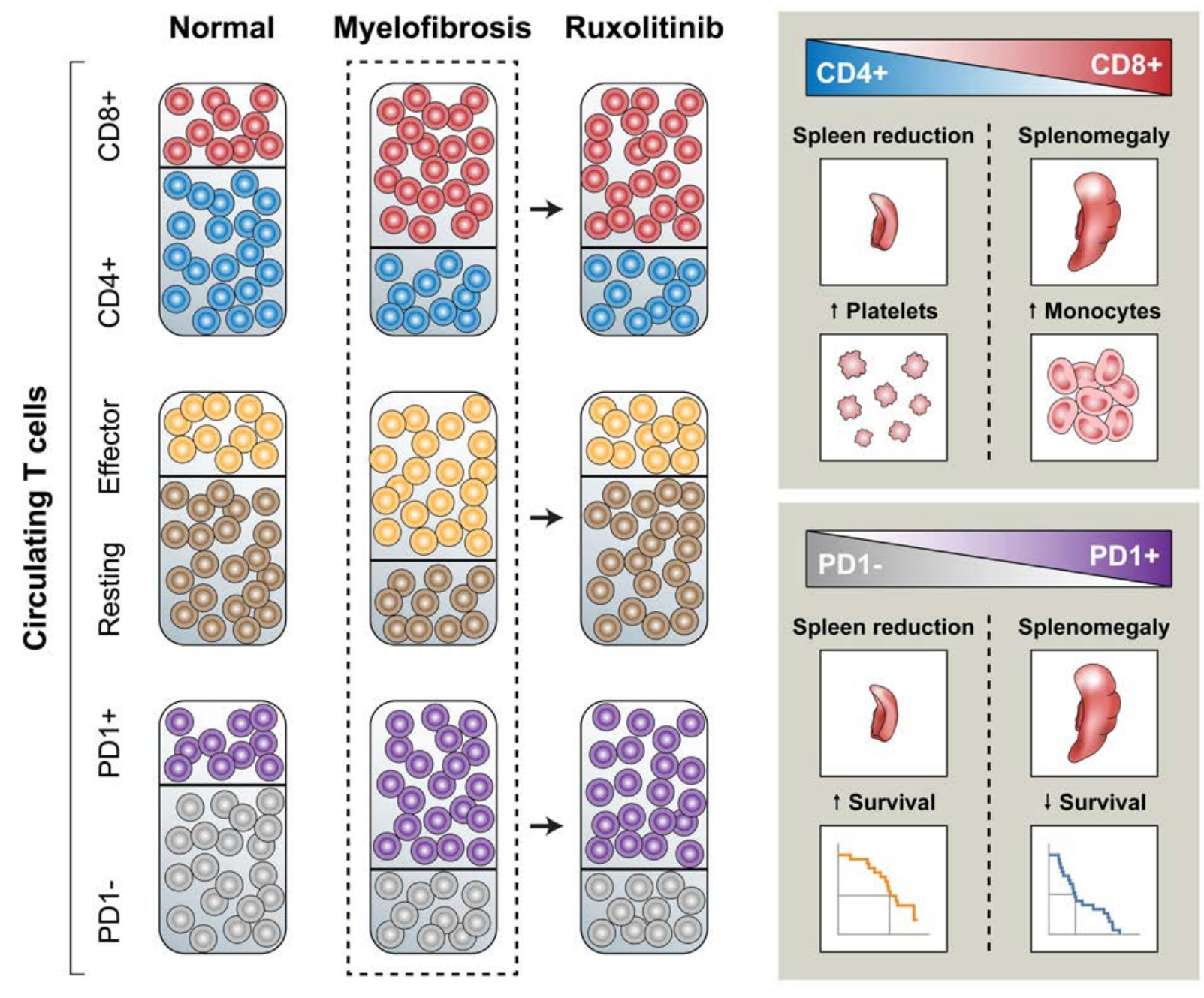

Figure 8. Schematic representation of the circulating T-cell subset repertoire in patients with myelofibrosis at baseline and after treatment with ruxolitinib. The left panel summarizes flow cytometry data from 47 patients with myelofibrosis (MF) analyzed in this study. Prior to treatment, T cells are skewed towards effector subsets (middle) and PD1-expressing T cells are increased (bottom) compared to those in 28 age-matched normal donors. In addition, disease progression shifts T-cell subsets towards CD8 ${ }^{+}$phenotypes (top). Ruxolitinib treatment reverts the resting:effector T-cell ratio to normal (middle), but has little effect on CD4/CD8 subsets or percentage of PD1 ${ }^{+}$cells. The right panel summarizes correlations of differentiation subsets (top) and PD $1^{+}$fractions (bottom) with the clinical parameters at treatment baseline (dashed line). Increases in CD8/PD1-coexpressing subsets are associated with a lack of spleen response. In addition, a CD8-predominant T-cell repertoire is found in patients with monocytosis and low platelet counts, whereas abundance of PD1-overexpressing CD8 ${ }^{+} \mathrm{T}$ cells is predictive of poor overall survival. 
tinib treatment have been associated with improved infectionfree survival. ${ }^{39}$ However, while neoplastic myeloid cells are thought to be the main target of JAK inhibitors, ruxolitinib also exerts a potent effect on non-malignant immune cells. The present study is the first to demonstrate that long-term treatment with ruxolitinib repolarizes activated T cells in MF patients. This finding is in agreement with previous in vitro studies demonstrating decreased cytokine production in T cells from ruxolitinibtreated MF patients, ${ }^{11}$ and decreased activation, proliferation and function of $\mathrm{T}$ cells from normal individuals. ${ }^{9}$ In addition, our observation of decreased $\mathrm{CD} 4^{+}$cell subsets as a result of ruxolitinib therapy confirms the findings of a previous study that showed JAK inhibitor-induced decreases in the number and function of helper T cells. ${ }^{10}$ Similar suppressive effects of ruxolitinib have been observed in NK cells from MF patients. ${ }^{40}$

Importantly, we observed increased $\mathrm{PD}^{+}$fractions among all assessed subsets, indicating that $\mathrm{T}$ cells are functionally exhausted in MF. These findings are in agreement with a recent study that showed increased PDL1 expression on myeloid cells from patients with JAK2-mutated myeloproliferative neoplasms. ${ }^{19}$ Our findings also corroborate PD1 expression patterns previously reported in circulating $\mathrm{CD} 4^{+}$and $\mathrm{CD} 8^{+}$cells of patients with $\mathrm{MF}^{20}$ In the aforementioned study, however, lack of any T-cellspecific markers in the gating strategy employed makes the reported results difficult to interpret. In our study, we specifically analyzed $\mathrm{CD} 45^{+} / \mathrm{CD}^{+} / \alpha \beta^{+} \mathrm{T}$ cells and assessed PD1-expressing cell fractions across the different activation subsets. T-cell exhaustion is typically manifested by a progressive defect in production of interferon- $\gamma$, IL-2, and tumor necrosis factor; T cells incapable of releasing these cytokines have been implicated in promoting the differentiation of monocytes into fibrocytes. ${ }^{41}$ It remains to be established how T-cell dysfunction affects the population of neoplastic fibrocytes, which induce BM fibrosis in $M F^{42}$ Conversely, there was no significant difference in the expression of either PD1 in T cells or PDL1 in blasts of patients with newly diagnosed acute myeloid leukemia, chronic myelomonocytic leukemia or myelodysplastic syndromes, ${ }^{37,43,44}$ suggesting that the neoplastic clone in MF exerts stronger immunogenicity with a significantly dysfunctional capacity as compared with other myeloid mallignancies.

In our cohort of MF patients, monocytosis and thrombocythopenia were associated with a predominantly CD8 ${ }^{+} \mathrm{T}$-cell phenotype. In addition, high levels of $\mathrm{CD}^{+}$cells and increased $\mathrm{PD}^{+}$fractions within the CD8 compartment correlated with disease progression and poor outcome. Although, we did not observe that ruxolitinib altered the percentage of $\mathrm{PD} 1^{+} \mathrm{T}$ cells, the survival of ruxolitinib-treated patients with low PD1 levels was significantly improved, suggesting that $\mathrm{T}$-cell dysfunction is associated with a poor response to treatment with ruxolitinib. Remarkably, like in MF, in acute lymphoblastic leukemia, a disease in which the JAK-STAT pathway is often constitutively activated, ${ }^{45-47}$ low numbers of $\mathrm{PD}^{+} \mathrm{T}$ cells predicted an improved treatment outcome. Because the spleen is a $\mathrm{T}$-cell reservoir, and spleen size correlated with $\mathrm{PD} 1^{+}$fractions, whereas a low $\mathrm{CD}^{+} / \mathrm{PD} 1^{+} \mathrm{T}$-cell percent correlated with a favorable response to ruxolitinib treatment, it is likely that T-cell exhaustion plays a role in the pathogenesis of MF and the response to JAK-inhibitor treatment.

Collectively, our data suggest that both cytotoxic and helper T cells in MF are overly activated and harbor increased $\mathrm{PD} 1^{+}$fractions. Long-term JAK inhibition reverses terminal T-cell overactivation; nonetheless, high levels of PD1-expressing $\mathrm{CD}^{+} \mathrm{T}$ cells result in poor survival. A further in-depth analysis of the innate immune system, including the heterogeneous T-cell populations and their interaction with the MF neoplastic myeloid cells, is warranted.

\section{Disclosures}

$S V$ receives research funding from Incyte Corporation, Wilmington, DE, USA. The remaining authors declare that they have no competing financial interests.

\section{Contributions}

$I V$ analyzed and interpreted data, performed the statistical analyses, created the figures, and wrote the manuscript; SP analyzed and interpreted data; SP and TM carried out the experiments; GMNG performed the statistical analyses; SV directed the project, supervised the study, and treated the patients included in the study; and ZE conceived, designed and supervised the study, interpreted data, and wrote the manuscript. All authors provided critical feedback and helped to develop the final manuscript.

\section{Acknowledgments}

The authors thank the Hanns A. Pielenz Foundation for financial support. The authors acknowledge Helen T. Chifotides for scientific editing assistance. This work was performed in part in the Flow Cytometry and Cellular Imaging Core Facility and used the Biostatistics Resource Group; both are supported by the National Cancer Institute, National Institutes of Health under award number P30 CA016672.

\section{References}

1. Barosi G. An immune dysregulation in MPN. Curr Hematol Malig Rep. 2014;9(4):331-339.

2. Hasselbalch HC, Bjorn ME. MPNs as inflammatory diseases: the evidence, consequences, and perspectives. Mediators Inflamm. 2015;2015:102476.

3. Vainchenker W, Kralovics R. Genetic basis and molecular pathophysiology of classical myeloproliferative neoplasms. Blood. 2017;129(6):667-679.

4. Hasselbalch HC. The role of cytokines in the initiation and progression of myelofibrosis. Cytokine Growth Factor Rev. 2013; 24(2):133-145.

5. Veletic I, Manshouri T, Newberry KJ, Garnett J, Verstovsek S, Estrov Z. Pentraxin3 plasma levels correlate with tumour burden and overall survival in patients with primary myelofibrosis. $\mathrm{Br} \mathrm{J}$ Haematol.
2018;185(2):382-386.

6. Villarino AV, Kanno Y, O'Shea JJ. Mechanisms and consequences of Jak-STAT signaling in the immune system. Nat Immunol. 2017;18(4):374-384.

7. Perner F, Perner C, Ernst T, Heidel FH. Roles of JAK2 in aging, inflammation, hematopoiesis and malignant transformation. Cells. 2019;8(8):854.

8. Elli EM, Borate C, Mendicino F, Palandri F, Palumbo GA. Mechanisms underlying the anti-inflammatory and immunosuppressive activity of ruxolitinib. Front Oncol. 2019;9:1186.

9. Heine A, Held SAE, Daecke SN, et al. The JAK-inhibitor ruxolitinib impairs dendritic cell function in vitro and in vivo. Blood. 2013;122(7):1192-1202.

10. Yajnanarayana SP, Stuebig T, Cornez I, et al. JAK1/2 inhibition impairs $T$ cell function invitro and in patients with myeloprolifera- tive neoplasms. Br J Haematol. 2015; 169(6):824-833.

11. Keohane C, Kordasti S, Seidl T, et al. JAK inhibition induces silencing of $\mathrm{T}$ helper cytokine secretion and a profound reduction in $\mathrm{T}$ regulatory cells. $\mathrm{Br} \mathrm{J}$ Haematol. 2015;171(1):60-73.

12. Holmstrom $\mathrm{MO}$, Riley $\mathrm{CH}$, Svane IM, Hasselbalch HC, Andersen MH. The CALR exon 9 mutations are shared neoantigens in patients with CALR mutant chronic myeloproliferative neoplasms. Leukemia. 2016; 30(12):2413-2416.

13. Holmstrom MO, Hjortso MD, Ahmad SM, et al. The JAK2V617F mutation is a target for specific $\mathrm{T}$ cells in the JAK2V617F-positive myeloproliferative neoplasms. Leukemia. 2017;31(2):495-498.

14. Holmstroem MO, Riley CH, Skov V, Svane IM, Hasselbalch HC, Andersen MH. Spontaneous $\mathrm{T}$-cell responses against the 
immune check point programmed-death-ligand 1 (PD-L1) in patients with chronic myeloproliferative neoplasms correlate with disease stage and clinical response. Oncoimmunology. 2018;7(6):e1433521.

15. Jorgensen MA, Holmstrom MO, Martinenaite E, Riley CH, Hasselbalch HC, Andersen MH. Spontaneous T-cell responses against Arginase-1 in the chronic myeloproliferative neoplasms relative to disease stage and type of driver mutation. Oncoimmunology. 2018;7(9):e1468957.

16. Blank CU, Haining WN, Held W, et al. Defining 'T cell exhaustion'. Nat Rev Immunol. 2019;19(11):665-674.

17. Sharma P, Allison JP. The future of immune checkpoint therapy. Science. 2015;348(6230): 56-61.

18. Sharpe AH, Pauken KE. The diverse functions of the PD1 inhibitory pathway. Nat Rev Immunol. 2018;18(3):153-167.

19. Prestipino A, Emhardt AJ, Aumann K, et al. Oncogenic JAK2(V617F) causes PD-L1 expression, mediating immune escape in myeloproliferative neoplasms. Sci Transl Med. 2018;10(429):eaam7729.

20. Wang J-C, Chen C, Kundra A, et al. Programmed cell death receptor (PD-1) ligand (PD-L1) expression in Philadelphia chromosome-negative myeloproliferative neoplasms. Leuk Res. 2019;79:52-59.

21. Green MR, Monti S, Rodig SJ, et al. Integrative analysis reveals selective 9p24.1 amplification, increased PD-1 ligand expression, and further induction via JAK2 in nodular sclerosing Hodgkin lymphoma and primary mediastinal large B-cell lymphoma. Blood. 2010;116(17):3268-3277.

22. Keenan TE, Burke KP, Van Allen EM. Genomic correlates of response to immune checkpoint blockade. Nat Med. 2019;25(3): 389-402.

23. Shin DS, Zaretsky JM, Escuin-Ordinas H, et al. Primary resistance to PD-1 blockade mediated by JAK1/2 mutations. Cancer Discov. 2017;7(2):188-201.

24. Verstovsek S, Kantarjian H, Mesa RA, et al. Safety and efficacy of INCB018424, a JAK1 and JAK2 inhibitor, in myelofibrosis. N Engl J Med. 2010;363(12):1117-1127.

25. Vardiman JW, Thiele J, Arber DA, et al. The 2008 revision of the World Health Organization (WHO) classification of myeloid neoplasms and acute leukemia: rationale and important changes. Blood. 2009;114(5):937-951.

26. Verstovsek S, Mesa RA, Gotlib J, et al. A double-blind, placebo-controlled trial of ruxolitinib for myelofibrosis. N Engl J Med. 2012;366(9):799-807.

27. Verstovsek S, Mesa RA, Gotlib J, et al. Long term treatment with ruxolitinib for patients with myelofibrosis: 5 -year update from the randomized, double-blind, placebo-controlled, phase 3 COMFORT-I trial. J Hematol Oncol. 2017;10(1):55

28. Cervantes F, Dupriez B, Passamonti F, et al. Improving survival trends in primary myelofibrosis: an international study. J Clin Oncol. 2012;30(24):2981-2987.

29. Chen DS, Mellman I. Elements of cancer immunity and the cancer-immune set point. Nature. 2017;541(7637):321-330.

30. Boiocchi L, Espinal-Witter R, Geyer JT, et al Development of monocytosis in patients with primary myelofibrosis indicates an accelerated phase of the disease. Mod Pathol. 2013;26(2):204-212

31. Cardoso EM, Esgalhado AJ, Patrao L, et al. Distinctive CD8(+) T cell and MHC class I signatures in polycythemia vera patients. Ann Hematol. 2018;97(9):1563-1575.

32. Brutkiewicz RR. Cell signaling pathways that regulate antigen presentation. J Immunol. 2016;197(8):2971-2979.

33. Romano M, Sollazzo D, Trabanelli S, et al Mutations in JAK2 and Calreticulin genes are associated with specific alterations of the immune system in myelofibrosis. Oncoimmunology. 2017;6(10):e1345402.

34. Le Dieu R, Taussig DC, Ramsay AG, et al. Peripheral blood T cells in acute myeloid leukemia (AML) patients at diagnosis have abnormal phenotype and genotype and form defective immune synapses with AML blasts. Blood. 2009:114(18):3909-3916.

35. Rey J, Fauriat C, Kochbati E, et al. Kinetics of cytotoxic lymphocytes reconstitution after induction chemotherapy in elderly AML patients reveals progressive recovery of normal phenotypic and functional features in NK cells. Front Immunol. 2017:8:64.

36. Vidriales MB, Orfao A, Lopezberges MC, et al. Lymphoid subsets in acute myeloid leukemias - increased number of cells with NK phenotype and normal T-cell distribu- tion. Ann Hematol. 1993:67(5):217-222.

37. Schnorfeil FM, Lichtenegger FS, Emmerig K, et al. T cells are functionally not impaired in AML: increased PD-1 expression is only seen at time of relapse and correlates with a shift towards the memory T cell compartment. J Hematol Oncol. 2015;8(93):93.

38. O'Donnell JS, Teng MWL, Smyth MJ. Cancer immunoediting and resistance to $\mathrm{T}$ cell-based immunotherapy. Nat Rev Clin Oncol. 2019;16(3):151-167.

39. Polverelli N, Palumbo GA, Binotto G, et al Epidemiology, outcome, and risk factors for infectious complications in myelofibrosis patients receiving ruxolitinib: a multicenter study on 446 patients. Hematol Oncol. 2018;36(3):561-569

40. Schoenberg K, Rudolph J, Vonnahme M, et al. JAK inhibition impairs NK cell function in myeloproliferative neoplasms. Cancer Res. 2015;75(11):2187-2199.

41. Niedermeier M, Reich B, Gomez MR, et al CD4(+) T cells control the differentiation of $\mathrm{Gr} 1(+)$ monocytes into fibrocytes. Proc Natl Acad Sci U S A. 2009;106(42):17892-17897.

42. Verstovsek S, Manshouri T, Pilling D, et al. Role of neoplastic monocyte-derived fibrocytes in primary myelofibrosis. J Exp Med. 2016;213(9):1723-1740.

43. Yang H, Bueso-Ramos C, DiNardo C, et al. Expression of PD-L1, PD-L2, PD-1 and CTLA4 in myelodysplastic syndromes is enhanced by treatment with hypomethylating agents. Leukemia. 2014;28(6):12801288

44. Kronig H, Kremmler L, Haller B, et al Interferon-induced programmed death-ligand 1 (PD-L1/B7-H1) expression increases on human acute myeloid leukemia blast cells during treatment. Eur J Haematol. 2014; 92(3):195-203

45. Vainchenker W, Constantinescu SN. JAK/STAT signaling in hematological malignancies. Oncogene. 2013;32(21):2601-2613.

46. Yang K, Xu J, Liu OH, Li J, Xi YF. Expression and significance of CD47, PD1 and PDL1 in T-cell acute lymphoblastic lymphoma/ leukemia. Pathol Res Pract. 2019; 215(2):265271.

47. Hohtari H, Bruck O, Blom S, et al. Immune cell constitution in bone marrow microenvironment predicts outcome in adult ALL. Leukemia. 2019;33(7):1570-1582. 